JOURNAL OF

SYMPLECTIC GEOMETRY

Volume 10, Number 3, 389-422, 2012

\title{
SPIN-QUANTIZATION COMMUTES WITH REDUCTION
}

\author{
PAUl-Emile Paradan
}

In this paper, we prove that the "quantization commutes with reduction" phenomenon of Guillemin and Sternberg [12] applies in the context of the metaplectic correction.

\section{Contents}

1. Introduction

2. Spin-quantization of compact Hamiltonian $K$-manifolds 393

2.1. Spin-quantization: definitions 393

2.2. Functorial properties 396

2.3. Spin-quantization of coadjoint orbits. $\quad 397$

2.4. Spin-prequantization commutes with reduction 398

3. Spin-quantization commutes with reduction 401

3.1. Elliptic and transversally elliptic symbols $\quad 402$

3.2. Localization of the Riemann-Roch character $\quad 404$

3.3. Induction formulas $\quad 406$

3.4. The torus case 407

3.4.1. First case: $\mu$ is a regular value of $\Phi \quad 408$

3.4.2. Second case: $\mu$ is a not (necessarily) a regular value of $\Phi \quad 408$

3.4.3. Proof of Theorem $2.10 \quad 409$

4. Proof of Theorem $3.1 \quad 410$

4.1. Proofs of Propositions 4.1 and $4.2 \quad 411$

1991 Mathematics Subject Classification: 58F06, 57S15, 19L47. 


\subsection{Computation of the multiplicities when $\sigma=\mathfrak{t}_{+}^{*} \quad 414$ 4.3. Computation of the multiplicities when $\sigma \neq \mathfrak{t}_{+}^{*} \quad 416$ References

\section{Introduction}

Let $K$ be a compact connected Lie group with Lie algebra $\mathfrak{k}$. An Hamiltonian $\mathrm{K}$-manifold $(M, \omega, \Phi)$ is Spin-prequantized if $M$ carries an equivariant $\operatorname{Spin}^{c}$ structure $P$ with determinant line bundle being a Kostant-Souriau line bundle over $(M, 2 \omega, 2 \Phi)$. Let $\mathcal{D}_{P}$ be the $\operatorname{Spin}^{c}$ Dirac operator attached to $P$, where $M$ is oriented by its symplectic form. The Spin quantization of $(M, \omega, \Phi)$ corresponds to the equivariant index of the elliptic operator $\mathcal{D}_{P}$, and is denoted

$$
\mathcal{Q}_{\text {spin }}^{K}(M) \in R(K) .
$$

Let $\widehat{A}(M)(X)$ be the equivariant $\hat{\mathrm{A}}$-genus class: it is an equivariant analytic function from a neighborhood of $0 \in \mathfrak{k}$ with value in the algebra of differential forms on $M$. The Atiyah-Segal-Singer index theorem [6, Theorem 8.2] tell us that

$$
\mathcal{Q}_{\text {spin }}^{K}(M)\left(e^{X}\right):=\left(\frac{\mathrm{i}}{2 \pi}\right)^{\frac{\operatorname{dim} M}{2}} \int_{M} \mathrm{e}^{\mathrm{i}(\omega+\langle\Phi, X\rangle)} \widehat{A}(M)(X)
$$

for $X \in \mathfrak{k}$ small enough. It shows in particular that $\mathcal{Q}_{\text {spin }}^{K}(M) \in R(K)$ does not depend of the choice of the Spin-prequantum data.

This notion of Spin-quantization is closely related to the notion of metaplectic correction. Suppose that $(M, \omega, \Phi)$ carries a Kostant-Souriau line bundle $L_{\omega}$, and that the bundle of half-forms $\kappa_{J}^{1 / 2}$ associated to an invariant almost complex structure $J$ is well defined. In this case, $(M, \omega, \Phi)$ is Spin-prequantized by the $\operatorname{Spin}^{c}$-structure defined by $J$ and twisted by the line bundle $L_{\omega} \otimes \kappa_{J}^{1 / 2}$. The crucial point here is that the corresponding Spin-quantization of $(M, \omega, \Phi)$ does not depend of the choice of the almost complex structure. Note that the existence of the bundle of half-form $\kappa_{J}^{1 / 2}$ is equivalent to the existence of a Spin structure on $M[\mathbf{1 6}]$.

The purpose of this paper is to compute geometrically the multiplicities of $\mathcal{Q}_{\text {spin }}^{K}(M) \in R(K)$ in a way similar to the famous "quantization commutes with reduction" phenomenon of Guillemin-Sternberg $[\mathbf{1 2}, \mathbf{1 4}, \mathbf{1 8}, \mathbf{1 9}, \mathbf{2 1}, \mathbf{2 4}, \mathbf{2 7}-\mathbf{2 9}]$. This question was first addressed in the work of Cannas-Karshon-Tolman [9] and Vergne [28] in the case of a circle action. The non-abelian group action case was first studied by Jeffrey-Kirwan [14] and by the author $[\mathbf{2 2}]$, but both papers made fairly strong assumptions: in [14] they suppose that $0 \in \mathfrak{k}^{*}$ has a big enough neighborhood of regular 
values of the moment map, and in [22] one asks that the infinitesimal stabilizers of the $K$-action are abelian. In this paper, we obtain a "quantization commutes with reduction" theorem, which holds in the general case. Note that C. Teleman also obtained some results [26, Proposition 3.10] in the algebraic setting.

The striking difference with the standard Guillemin-Sternberg phenomenon is the rho shift that we explain now. Let $T$ be a maximal torus of $K$ with Lie algebra $\mathfrak{t} \subset \mathfrak{k}$. Let $\mathfrak{t}_{+}^{*} \subset \mathfrak{t}^{*}$ be the closed Weyl chamber. We will look at $\mathfrak{t}_{+}^{*}$ as a disjoint union of its open faces, the maximal one being its interior $\left(\mathfrak{t}_{+}^{*}\right)^{\mathrm{o}}$. Let $\rho \in\left(\mathfrak{t}_{+}^{*}\right)^{\mathrm{o}}$ be the half sum of the positive roots. At each open face $\tau$ of $\mathfrak{t}_{+}^{*}$, we associate the term $\rho_{\tau}$, which is the half sum of the positive roots which are orthogonal to $\tau$. We note that $\rho-\rho_{\tau} \in \tau$ is equal to the orthogonal projection of $\rho$ on $\tau$.

For any $\xi \in \mathfrak{t}_{+}^{*}$ and any face $\tau$ containing $\xi$ in its closure, we consider the shifted symplectic reduction

$$
M_{\xi}^{\tau}:=\Phi^{-1}\left(\xi+\rho-\rho_{\tau}\right) / K_{\tau}
$$

where $K_{\tau}$ is the common stabilizer of points in $\tau$. Note that $\xi+\rho-\rho_{\tau} \in \tau$ when $\xi \in \bar{\tau}$.

We are particularly interested to the smallest face $\sigma$ of the Weyl chamber so that the Kirwan polytope $\Delta(M):=\Phi(M) \cap \mathfrak{t}_{+}^{*}$ is contained in the closure of $\sigma$. It is not hard to see that the Spin-prequantum data on $(M, \omega, \Phi)$ descents to the shifted symplectic reduction $M_{\mu}^{\sigma}$ when $\mu$ is a dominant weight belonging to $\bar{\sigma}$. Then $\mathcal{Q}_{\text {spin }}\left(M_{\mu}^{\sigma}\right) \in \mathbb{Z}$ is naturally defined when $\mu+\rho-\rho_{\sigma}$ is a regular value of the moment map. In general, the number $\mathcal{Q}_{\text {spin }}\left(M_{\mu}^{\sigma}\right)$ is defined by shift-desingularization (see Section 2.4).

By definition $\mathcal{Q}_{\text {spin }}\left(M_{\mu}^{\sigma}\right)$ vanishes when $\mu+\rho-\rho_{\sigma} \notin \Delta(M)$, but in fact we can strengthen this vanishing property: $\mathcal{Q}_{\text {spin }}\left(M_{\mu}^{\sigma}\right)=0$ if $\mu+\rho-\rho_{\sigma}$ does not belong to the relative interior of the Kirwan polytope $\Delta(M)$.

Recall that the irreducible representations $V_{\mu}^{K}$ of $K$ are parametrized by their highest weight $\mu \in \widehat{K} \subset \mathfrak{t}_{+}^{*}$.

The main result of this paper is the following:

Theorem 1.1. Let $(M, \omega, \Phi)$ be a compact Spin-prequantized Hamiltonian $K$-manifold. Let $\sigma$ be the smallest face of the Weyl chamber so that $\Delta(M) \subset$ $\bar{\sigma}$. We have

$$
\mathcal{Q}_{\text {spin }}^{K}(M)=\sum_{\mu \in \widehat{K} \cap \bar{\sigma}} \mathcal{Q}_{\text {spin }}\left(M_{\mu}^{\sigma}\right) V_{\mu}^{K}
$$

Let us give some ideas about the proof. The representation $V_{\mu}^{K}$ is equal to the Spin-quantization of the coadjoint orbit $\mathcal{O}_{\mu}:=K \cdot(\mu+\rho)$. Then the 
shifting trick tells us that the multiplicity $\mathrm{m}_{\mu}$ of $V_{\mu}^{K}$ in $\mathcal{Q}_{\text {spin }}^{K}(M)$ is equal to

$$
\left[\mathcal{Q}_{\text {spin }}^{K}(M) \otimes\left(V_{\mu}^{K}\right)^{*}\right]^{K}=\left[\mathcal{Q}_{\text {spin }}^{K}\left(M \times \overline{\mathcal{O}}_{\mu}\right)\right]^{K}
$$

where $\overline{\mathcal{O}}_{\mu}$ is the coadjoint orbit with the opposite symplectic structure. As we did in $[\mathbf{2 1}, \mathbf{2 2}]$, we study the expression $\left[\mathcal{Q}_{\text {spin }}^{K}\left(M \times \overline{\mathcal{O}}_{\mu}\right)\right]^{K}$ by localizing the Riemann-Roch character on the critical points of the square of the moment map

$$
\Phi_{\mu}: M \times \overline{\mathcal{O}}_{\mu} \rightarrow \mathfrak{k}^{*}
$$

Here our treatment differs depending on whether the Kirwan polytope $\Delta(M)$ intersects the interior of the Weyl chambers or not (i.e., $\sigma=\mathfrak{t}_{+}^{*}$ or not).

When $\sigma=\mathfrak{t}_{+}^{*}$, we show that the multiplicity $\mathrm{m}_{\mu}$ is calculated using the Riemann-Roch character localized near the zero-level set of the moment map $\Phi_{\mu}$. This case is (more or less) treated in [22].

The heart of this paper is when we work out the case $\sigma \neq \mathfrak{t}_{+}^{*}$. We have $\Phi_{\mu}^{-1}(0)=\emptyset$, but we show how to compute $\mathrm{m}_{\mu}$ using the Riemann-Roch character localized near

$$
K \cdot\left(N^{\rho_{\sigma}} \cap \Phi_{\mu}^{-1}\left(-\rho_{\sigma}\right)\right) .
$$

Here $N^{\rho_{\sigma}}$ denotes the submanifold of $N=M \times \mathcal{O}_{\mu}$ where the infinitesimal action of $\rho_{\sigma}$ vanishes.

Notations. Throughout the paper, $K$ will denote a compact connected Lie group, and $\mathfrak{k}$ its Lie algebra. We let $T$ be a maximal torus in $K$, and $\mathfrak{t}$ be its Lie algebra. The integral lattice $\wedge \subset \mathfrak{t}$ is defined as the kernel of $\exp : \mathfrak{t} \rightarrow T$, and the real weight lattice $\wedge^{*} \subset \mathfrak{t}^{*}$ is defined by $: \wedge^{*}:=\operatorname{hom}(\wedge, 2 \pi \mathbb{Z})$. Every $\mu \in \wedge^{*}$ defines a one-dimensional T-representation, denoted $\mathbb{C}_{\mu}$, where $t=$ $\exp (X)$ acts by $t^{\mu}:=\mathrm{e}^{\mathrm{i}\langle\mu, X\rangle}$. We fix a positive Weyl chamber $\mathfrak{t}_{+}^{*} \subset \mathfrak{t}^{*}$. For any dominant weight $\mu \in \widehat{K}:=\wedge^{*} \cap \mathfrak{t}_{+}^{*}$, we denote by $V_{\mu}^{K}$ the irreducible representation with highest weight $\mu$. We denote $R(K)$ the representation ring of $K$. We denote $R^{-\infty}(K):=\operatorname{hom}_{\mathbb{Z}}(R(K), \mathbb{Z})$ its dual. An element $E \in R^{-\infty}(K)$ can be represented as an infinite sum $E=\sum_{\mu \in \widehat{K}} \mathrm{~m}_{\mu} V_{\mu}^{K}$, with $\mathrm{m}_{\mu} \in \mathbb{Z}$. The multiplicity $\mathrm{m}_{0}$ of the trivial representation is denoted $[E]^{K}$. If $H$ is a closed subgroup of $K$, we have the induction map $\operatorname{Ind}_{H}^{K}: R^{-\infty}(H) \rightarrow$ $R^{-\infty}(K)$ which is the dual of the restriction morphism $R(K) \rightarrow R(H)$. We see that $\left[\operatorname{Ind}_{H}^{K}(E)\right]^{K}=[E]^{H}$.

When $K$ acts on a set $X$, the stabilizer subgroup of an element $x \in X$ is denoted $K_{x}:=\{k \in K \mid k \cdot x=x\}$. The Lie algebra of $K_{x}$ is denoted $\mathfrak{k}_{x}$. 


\section{Spin-quantization of compact Hamiltonian $K$-manifolds}

Let $M$ be a compact Hamiltonian $K$-manifold with symplectic form $\omega$ and moment map $\Phi: M \rightarrow \mathfrak{k}^{*}$ characterized by the relation

$$
\iota\left(X_{M}\right) \omega=-d\langle\Phi, X\rangle, \quad X \in \mathfrak{k},
$$

where $X_{M}(m):=\left.\frac{d}{d t}\right|_{t=0} \mathrm{e}^{-t X} \cdot m$ is the vector field on $M$ generated by $X \in \mathfrak{k}$.

In the Kostant-Souriau framework $\left[\mathbf{1 5}, \mathbf{2 5}\right.$ ], a Hermitian line bundle $L_{\omega}$ with an invariant Hermitian connection $\nabla$ is a prequantum line bundle over $(M, \omega, \Phi)$ if

$$
\mathcal{L}(X)-\nabla_{X_{M}}=\mathrm{i}\langle\Phi, X\rangle \text { and } \nabla^{2}=-\mathrm{i} \omega,
$$

for every $X \in \mathfrak{k}$. Here $\mathcal{L}(X)$ is the infinitesimal action of $X \in \mathfrak{k}$ on the sections of $L_{\omega} \rightarrow M$. $\left(L_{\omega}, \nabla\right)$ is also called a Kostant-Souriau line bundle. Remark that conditions (2.2) imply, via the equivariant Bianchi formula, the relation $(2.1)$.

2.1. Spin-quantization: definitions. Let $N$ be a compact even dimensional Riemannian manifold, and let $\mathrm{Cl}(N)$ be its Clifford bundle. A Spin $^{c}$ structure $P$ on $N$ defines an irreducible Clifford bundle $\mathcal{S}_{P} \rightarrow N$ [6, Section 3.3]. If $P$ and $P^{\prime}$ are two $\operatorname{Spin}^{c}$ structures on $N$, then we have $\mathcal{S}_{P^{\prime}} \simeq \mathcal{S}_{P} \otimes \mathbb{L}_{P, P^{\prime}}$ where $\mathbb{L}_{P, P^{\prime}}$ is a line bundle on $N$ defined by the relation

$$
\mathbb{L}_{P, P^{\prime}}:=\operatorname{hom}_{\mathrm{Cl}(N)}\left(\mathcal{S}_{P}, \mathcal{S}_{P^{\prime}}\right) .
$$

If $\mathcal{S} \rightarrow N$ is an irreducible Clifford bundle, then its complex dual $\mathcal{S}^{*} \rightarrow N$ is also an irreducible Clifford bundle.

Definition 2.1. The determinant line bundle of a $\operatorname{Spin}^{c}$ structure $P$ on $N$ is the line bundle $\operatorname{det}(P) \rightarrow N$ defined by the relation $\operatorname{det}(P):=$ $\operatorname{hom}_{\mathrm{Cl}(N)}\left(\mathcal{S}_{P}^{*}, \mathcal{S}_{P}\right)$.

If $P$ and $P^{\prime}$ are two $\operatorname{Spin}^{c}$ structures on $N$, we see that

$$
\begin{aligned}
\operatorname{det}\left(P^{\prime}\right) & \simeq \operatorname{hom}_{\mathrm{Cl}(N)}\left(\mathcal{S}_{P}^{*} \otimes \mathbb{L}_{P, P^{\prime}}^{-1}, \mathcal{S}_{P} \otimes \mathbb{L}_{P, P^{\prime}}\right) \\
& \simeq \operatorname{det}(P) \otimes\left(\mathbb{L}_{P, P^{\prime}}\right)^{2} .
\end{aligned}
$$

On the other hand, we can twist a $\operatorname{Spin}^{c}$ structure $P$ on $N$ by a complex line bundle $\mathbb{L} \rightarrow N$ : its defines another $\operatorname{Spin}^{c}$ structure $P_{\mathbb{L}}$ such that $\mathcal{S}_{P_{\mathbb{L}}}=$ $\mathcal{S}_{P} \otimes \mathbb{L}$.

Let us come back to the situation of a $K$-Hamiltonian manifold $(M, \omega, \Phi)$. Let $J$ be any invariant almost complex structure on $M$, not necessarily compatible with the symplectic form $\omega$. Let

$$
R R_{J}^{K}(M,-)
$$


be the corresponding Riemann-Roch character $[\mathbf{2 1}]$. We consider the complex tangent bundle $(\mathrm{T} M, J)$ and its complex dual $\mathrm{T}_{\mathbb{C}}^{*} M:=\operatorname{hom}_{\mathbb{C}}(\mathrm{T} M, \mathbb{C})$. We consider the complex line bundle

$$
\kappa_{J}:=\operatorname{det} \mathrm{T}_{\mathbb{C}}^{*} M .
$$

If $(M, \omega, \Phi)$ is prequantized by $L_{\omega}$, a standard procedure (called the metaplectic correction in the geometric quantization literature) is to tensor $L_{\omega}$ by the bundle of half-forms $\kappa_{J}^{1 / 2}[\mathbf{3 0}]$. We may consider the equivariant index

$$
\mathcal{Q}_{J}^{K}(M):=\epsilon_{J} R R_{J}^{K}\left(M, L_{\omega} \otimes \kappa_{J}^{1 / 2}\right)
$$

where $\epsilon_{J}= \pm 1$ is the quotient of the orientations defined by $\omega$ and by $J$. In Proposition 2.3 we check that $\mathcal{Q}_{J}^{K}(M)$ has a meaning when the tensor product $\tilde{L}=L_{\omega} \otimes \kappa_{J}^{1 / 2}$ is well defined (even if neither $L_{\omega}$ nor $\kappa_{J}^{1 / 2}$ exist).

The almost complex structure $J$ defines a $\operatorname{Spin}^{c}$ structure $P_{J}$ on $M$ with determinant line bundle $\operatorname{det} P_{J}=\kappa_{J}^{-1}$ (see $[\mathbf{1 6}, \mathbf{2 2}]$ ). If we twist the $\operatorname{Spin}^{c}$ structure $P_{J}$ by any complex line bundle $\mathbb{L}$ we get a Spin ${ }^{c}$ structure $P_{J, \mathbb{L}}$ with determinant line bundle

$$
\operatorname{det}\left(P_{J, \mathbb{L}}\right)=\kappa_{J}^{-1} \otimes \mathbb{L}^{2}
$$

We make the following basic observation.

Proposition 2.2. Let $(M, \omega, \Phi)$ be a Hamiltonian $K$-manifold. The following assertions are equivalent:

(a) For any invariant complex structure $J$ there exists a $K$-equivariant line bundle $\tilde{L}$ such that $\kappa_{J}^{-1} \otimes \tilde{L}^{2}$ is a prequantum line bundle over $(M, 2 \omega, 2 \Phi)$.

(b) There exist an invariant complex structure $J$ and a $K$-equivariant line bundle $\tilde{L}$ such that $\kappa_{J}^{-1} \otimes \tilde{L}^{2}$ is a prequantum line bundle over $(M, 2 \omega, 2 \Phi)$.

(c) There exists an equivariant Spin $^{c}$ structure $P$ such that its determinant line bundle $\operatorname{det}(P)$ is a prequantum line bundle over $(M, 2 \omega, 2 \Phi)$.

When the previous assertions holds, we says that $(M, \omega, \Phi)$ is Spinprequantized, either by the $\operatorname{Spin}^{c}$-structure $P$, or by the data $(J, \tilde{L})$.

Proposition 2.3. Let $(M, \omega, \Phi)$ be a Spin-prequantized Hamiltonian $K$ manifold. The equivariant index $\mathcal{Q}_{J}^{K}(M):=\epsilon_{J} R R_{J}^{K}(M, \tilde{L})$ does not depend of the choice of the Spin-prequantum data $(J, \tilde{L})$. In fact $\mathcal{Q}_{J}^{K}(M)$ coincides with the equivariant index of the $\operatorname{Spin}^{c}$ Dirac operator $\mathcal{D}_{P}$ attached to the Spin ${ }^{c}$-structure $P$.

Definition 2.4. Let $(M, \omega, \Phi)$ be a Spin-prequantized Hamiltonian $K$ manifold. The Spin-quantization of $(M, \omega, \Phi)$ is defined as the equivariant index $\mathcal{Q}_{J}^{K}(M)$, and is denoted

$$
\mathcal{Q}_{\text {spin }}^{K}(M) \in R(K) \text {. }
$$


Proof of Propositions 2.2 and 2.3. We have obviously $a) \Longrightarrow b$ ), and we get $b) \Longrightarrow c$ ) by taking the $\operatorname{Spin}^{c}$ structure $P_{J, \tilde{L}}$. Let us prove $\left.c\right) \Longrightarrow a$ ).

Let $P$ be a $\operatorname{Spin}^{c}$-structure on $M$ such that its determinant line bundle $\operatorname{det}(P)$ is a prequantum line bundle over $(M, 2 \omega)$. Let $\mathcal{S}_{P}$ be the corresponding bundle of spinors. Let $P_{J}$ and $\mathcal{S}_{J}$ be respectively the associated $\mathrm{Spin}^{c}$ structure and the bundle of spinors on $M$ associated to an invariant almost complex structure $J$ on $M$. Since $\mathcal{S}_{P}, \mathcal{S}_{J}$ are irreducible clifford modules, we have

$$
\mathcal{S}_{P} \simeq \mathcal{S}_{J} \otimes \tilde{L}
$$

where $\tilde{L}$ is the line bundle defined by $\tilde{L}:=\operatorname{hom}_{\mathrm{Cl}(M)}\left(\mathcal{S}_{J}, \mathcal{S}_{P}\right)$. From $(2.5)$ we get that the line bundle

$$
\begin{aligned}
\operatorname{det}(P) & \simeq \operatorname{det}\left(P_{J}\right) \otimes \tilde{L}^{2} \\
& \simeq \kappa_{J}^{-1} \otimes \tilde{L}^{2}
\end{aligned}
$$

is a prequantum line bundle over $(M, 2 \omega, 2 \Phi)$.

Let $P$ be the $\operatorname{Spin}^{c}$ structure attached to a data $(J, \tilde{L})$. The symplectic orientation on $M$ defines a decomposition on the bundle of spinors, $\mathcal{S}_{P}=\mathcal{S}_{P}^{+} \oplus \mathcal{S}_{P}^{-}$, and the corresponding $\operatorname{Spin}^{c}$ Dirac operator $\mathcal{D}_{P}$ maps $\Gamma\left(\mathcal{S}_{P}^{+}\right)$ to $\Gamma\left(\mathcal{S}_{P}^{-}\right)$.

On the other hand, the almost complex structure on $M$ gives the decomposition $\wedge \mathrm{T}^{*} M \otimes \mathbb{C}=\oplus_{i, j} \wedge^{i, j} \mathrm{~T}^{*} M$ of the bundle of differential form. The corresponding bundle of spinors is $\mathcal{S}_{J}:=\wedge^{0, \bullet} \mathrm{T}^{*} M$ and the complex orientation induces the splitting $\mathcal{S}_{J}=\mathcal{S}_{J}^{+} \oplus \mathcal{S}_{J}^{-}$with $\mathcal{S}_{J}^{+}:=\wedge^{0 \text {,even }} \mathrm{T}^{*} M$. The Dolbeault Dirac operator $\bar{\partial}_{\tilde{L}}+\bar{\partial}_{\tilde{L}}^{*}$ maps $\Gamma\left(\mathcal{S}_{J}^{ \pm} \otimes \tilde{L}\right)$ to $\Gamma\left(\mathcal{S}_{J}^{\mp} \otimes \tilde{L}\right)$, and the Riemann-Roch character $R R_{J}^{K}(M, \tilde{L})$ is defined as the equivariant index of the elliptic operator

$$
\bar{\partial}_{\tilde{L}}+\bar{\partial}_{\tilde{L}}^{*}: \Gamma\left(\mathcal{S}_{J}^{+} \otimes \tilde{L}\right) \longrightarrow \Gamma\left(\mathcal{S}_{J}^{-} \otimes \tilde{L}\right) .
$$

If $\epsilon_{J}= \pm 1$ is the quotient of the orientations defined by $\omega$ and by $J$, one has that

$$
\mathcal{S}_{P}^{ \pm}=\mathcal{S}_{J}^{ \pm \epsilon_{J}} \otimes \tilde{L} .
$$

Hence $\mathcal{Q}_{J}^{K}(M)=\epsilon_{J} R R_{J}^{K}(M, \tilde{L})$ is defined as the equivariant index of the Dolbeault Dirac operator $\bar{\partial}_{\tilde{L}}+\bar{\partial}_{\tilde{L}}^{*}$ viewed as an elliptic operator $\mathcal{D}_{\tilde{L}}^{+}$from $\Gamma\left(\mathcal{S}_{P}^{+}\right)$to $\Gamma\left(\mathcal{S}_{P}^{-}\right)$.

Finally, we know that $\operatorname{Index}^{K}\left(\mathcal{D}_{P}\right)=\operatorname{Index}^{K}\left(\mathcal{D}_{\tilde{L}}^{+}\right)$since the first-order elliptic operators $\mathcal{D}_{P}$ and $\mathcal{D}_{\tilde{L}}^{+}$have the same principal symbol [10].

In the remaining part of this paper, we find convenient to work with the following 
Definition 2.5. A Hamiltonian $K$-manifold $(M, \omega, \Phi)$ is Spin-prequantized by $\tilde{L}$ if there exists an invariant almost complex structure $J$ compatible with $\omega$ such that $\tilde{L}^{2} \otimes \kappa_{J}^{-1}$ is a Kostant-Souriau line bundle over $(M, 2 \omega, 2 \Phi)$.

We remark that $\varepsilon_{J}=1$ when $J$ is compatible with $\omega$. Moreover, the Riemann-Roch character $R R_{J}^{K}(M,-)$ does not depend [21] on the choice of the compatible invariant almost complex structure $J$ : we denote it simply by $R R^{K}(M,-)$.

Finally, when a Hamiltonian manifold $(M, \omega, \Phi)$ is Spin-prequantized by the line bundle $\tilde{L}$, its Spin-quantization is defined by

$$
\mathcal{Q}_{\text {spin }}^{K}(M):=R R^{K}(M, \tilde{L}) .
$$

2.2. Functorial properties. We summarize the functorial properties of $\mathcal{Q}_{\text {spin }}$ in the next

Proposition 2.6. - If $(M, \omega, \Phi)$ is a Spin-prequantized Hamiltonian $K$-manifold, and $H$ is a closed subgroup of $K$ then the restriction of $\mathcal{Q}_{\text {spin }}^{K}(M)$ to $H$ is equal to $\mathcal{Q}_{\text {spin }}^{H}(M)$.

- If $\left(M_{j}, \omega_{j}, \Phi_{j}\right)$ are Spin-prequantized Hamiltonian $K_{j}$-manifold, for $j=1,2$, then $M_{1} \times M_{2}$ is a Spin-prequantized Hamiltonian $K_{1} \times K_{2}$ manifold and

$$
\mathcal{Q}_{\text {spin }}^{K_{1} \times K_{2}}\left(M_{1} \times M_{2}\right)=\mathcal{Q}_{\text {spin }}^{K_{1}}\left(M_{1}\right) \otimes \mathcal{Q}_{\text {spin }}^{K_{2}}\left(M_{2}\right)
$$

in $R\left(K_{1} \times K_{2}\right) \simeq R\left(K_{1}\right) \otimes R\left(K_{2}\right)$.

- If $\left(M, \omega_{M}, \Phi_{M}\right)$ and $\left(N, \omega_{N}, \Phi_{N}\right)$ are Spin-prequantized Hamiltonian $K$-manifold, then $M \times N$ is a Spin-prequantized Hamiltonian $K$-manifold and

$$
\mathcal{Q}_{\text {spin }}^{K}(M \times N)=\mathcal{Q}_{\text {spin }}^{K}(M) \cdot \mathcal{Q}_{\text {spin }}^{K}(N),
$$

where $\cdot$ denotes the product in $R(K)$.

- A Spin-prequantization on $(M, \omega, \Phi)$ induces a Spin-prequantization on $\bar{M}:=(M,-\omega,-\Phi)$. The Spin-quantization of $\bar{M}$ corresponds to the dual of the Spin-quantization of $M$ :

$$
\mathcal{Q}_{\text {spin }}^{K}(\bar{M})=\left[\mathcal{Q}_{\text {spin }}^{K}(M)\right]^{*}
$$

Proof. The first three points are direct consequences of the functorial properties of the index map. Let us prove the last point. One see that if $(\tilde{L}, J)$ is a Spin-prequantum data for $M$ then $\left(\tilde{L}^{-1},-J\right)$ is a Spin-prequantum data 
for $\bar{M}$. Then we have for $X \in \mathfrak{k}$ small enough

$$
\begin{aligned}
\mathcal{Q}_{\text {spin }}^{K}(\bar{M})\left(\mathrm{e}^{X}\right) & =\frac{\left(\frac{\mathrm{i}}{2 \pi}\right)^{\frac{\operatorname{dim} M}{2}} \int_{\bar{M}} \mathrm{e}^{\mathrm{i}(-\omega-\langle\Phi, X\rangle)} \widehat{A}(M)(X)}{\left(\frac{\mathrm{i}}{2 \pi}\right)^{\frac{\operatorname{dim} M}{2}} \int_{M} \mathrm{e}^{\mathrm{i}(\omega+\langle\Phi, X\rangle) \widehat{A}(M)(X)}} \\
& =\frac{\mathcal{Q}_{\text {spin }}^{K}(M)\left(\mathrm{e}^{X}\right)}{}
\end{aligned}
$$

The relation [1] is due to the fact that the differential form $\widehat{A}(M)(X)$ has real coefficients, and that the quotient of the symplectic orientations on $M$ and $\bar{M}$ is $(-1)^{\frac{\operatorname{dim} M}{2}}$. Since $X \rightarrow \mathcal{Q}_{\text {spin }}^{K}(M)\left(\mathrm{e}^{X}\right)$ are analytic functions, the identity [2] shows that $\mathcal{Q}_{\text {spin }}^{K}(\bar{M})(k)=\overline{\mathcal{Q}_{\text {spin }}^{K}(M)(k)}$ for any $k \in K$. In other words the (virtual) representation $\mathcal{Q}_{\text {spin }}^{K}(\bar{M})$ corresponds to the dual of the (virtual) representation $\mathcal{Q}_{\text {spin }}^{K}(M)$.

2.3. Spin-quantization of coadjoint orbits. Let $\mu \in \widehat{K}$ be a dominant weight. Let us denote $K_{\mu}$ its stabilizer subgroup and $\mathfrak{k}_{\mu}$ its Lie algebra. Let us recall why the Lie algebra morphism $\mathrm{i} \mu: \mathfrak{k}_{\mu} \rightarrow \mathrm{i} \mathbb{R}$ integrates in a character $\chi_{\mu}$ of $K_{\mu}$. The group $K_{\mu}$, which is connected, decomposes as $K_{\mu}=\left[K_{\mu}, K_{\mu}\right] Z_{\mu}$ where $Z_{\mu}$ is the connected component of the center of $K_{\mu}$. For the maximal torus $T$, we have $T=T_{\mu} Z_{\mu}$ with $T_{\mu}=T \cap\left[K_{\mu}, K_{\mu}\right]=\exp \left(\mathfrak{t} \cap\left[\mathfrak{k}_{\mu}, \mathfrak{k}_{\mu}\right]\right)$. We note that $\mathrm{i} \mu: \mathfrak{t} \rightarrow \mathrm{i} \mathbb{R}$ integrates in a character $\chi_{\mu}^{T}$ of $T$ which is trivial on $T_{\mu}$ since $\left\langle\mu,\left[\mathfrak{k}_{\mu}, \mathfrak{k}_{\mu}\right]\right\rangle=0$. Hence, we can define the character $\chi_{\mu}$ as being trivial on $\left[K_{\mu}, K_{\mu}\right]$, and equal to $\chi_{\mu}^{T}$ on $Z_{\mu}$.

We denote by $\mathbb{C}_{\mu}$ the one-dimensional representation of $K_{\mu}$ associated to the character $\chi_{\mu}$. Let $\sigma$ be a face of the Weyl chamber such that $\mu \in \bar{\sigma}$ : hence the stabilizer subgroup $K_{\mu}$ contains $K_{\sigma}$. We still denote by $\mathbb{C}_{\mu}$ the induced one-dimensional representation of the group $K_{\sigma}$.

Let $\rho$ be half the sum of the positive roots, and let $\rho_{\sigma}$ be half the sum of the positive roots, which are orthogonal to $\sigma$. Note that $\rho-\rho_{\sigma}$ belongs to $\sigma$, hence $\mu+\rho-\rho_{\sigma}$ belongs also to $\sigma$ for any $\mu \in \bar{\sigma}$. The coadjoint orbit

$$
\mathcal{O}_{\mu}^{\sigma}:=K \cdot\left(\mu+\rho-\rho_{\sigma}\right) \simeq K / K_{\sigma}
$$

is Spin-prequantized by the compatible complex structure and the line bundle $\tilde{L}=K \times_{K_{\sigma}} \mathbb{C}_{\mu}$. We have

$$
\begin{aligned}
\mathcal{Q}_{\text {spin }}^{K}\left(\mathcal{O}_{\mu}^{\sigma}\right) & =R R^{K}\left(K / K_{\sigma}, K \times_{K_{\sigma}} \mathbb{C}_{\mu}\right) \\
& =V_{\mu}^{K}
\end{aligned}
$$

thanks to the Borel-Weil theorem. We know also that $\mathcal{Q}_{\text {spin }}^{K}\left(\overline{\mathcal{O}_{\mu}^{\sigma}}\right)=\left(V_{\mu}^{K}\right)^{*}$, where $\overline{\mathcal{O}_{\mu}^{\sigma}}$ be the coadjoint orbit $\mathcal{O}_{\mu}^{\sigma}$ with the opposite symplectic form (see Proposition 2.6). 
We have seen that the same irreducible representations $V_{\mu}^{K}$ can be realized as the Spin-quantization of the coadjoint orbits $\mathcal{O}_{\mu}^{\sigma}$ where $\sigma$ is a face of the Weyl chamber containing $\mu$ in its closure.

2.4. Spin-prequantization commutes with reduction. We consider first the case of a Hamiltonian $H$-manifold $(N, \omega, \Phi)$, not necessarily compact, which is Spin-prequantized by $\tilde{L}$. We suppose that 0 is a regular value of $\Phi$. Let $N_{0}:=\Phi^{-1}(0) / H$ be the orbifold reduced space with its canonical symplectic structure $\omega_{0}$.

Lemma 2.7. The orbifold line bundle $\tilde{\mathcal{L}}_{0}:=\left(\left.\tilde{L}\right|_{\Phi^{-1}(0)}\right) / H$ Spin-prequantizes $\left(N_{0}, \omega_{0}\right)$.

Proof. The fiber $\mathcal{Z}=\Phi^{-1}(0)$ is a smooth $H$-invariant submanifold of $N$. Let $\pi: \mathcal{Z} \rightarrow \mathcal{Z} / H=N_{0}$ be the projection. Recall that the symplectic structure $\omega_{0}$ on $N_{0}$ is defined by the relation $\pi^{*}\left(\omega_{0}\right)=\omega \mid \mathcal{Z}$. Let $L_{2 \omega}$ the Kostant-Souriau line bundle on $(N, 2 \omega, 2 \Phi)$ such that

$$
\tilde{L}^{2}=L_{2 \omega} \otimes \kappa_{J} \text {. }
$$

Here $J$ is a compatible invariant almost complex structure on $N$. We have $\left.\mathrm{T} N\right|_{\mathcal{Z}}=\mathrm{T} \mathcal{Z} \oplus J\left(\mathfrak{h}_{\mathcal{Z}}\right)$ where $\mathfrak{h}_{\mathcal{Z}} \subset \mathrm{T} \mathcal{Z}$ is the trivial bundle given by the infinitesimal action of $H$. Since $\mathrm{T} \mathcal{Z} \simeq \pi^{*}\left(\mathrm{~T} N_{0}\right) \oplus \mathfrak{h}_{\mathcal{Z}}$ we get

$$
\left.\mathrm{T} N\right|_{\mathcal{Z}} \simeq \pi^{*}\left(\mathrm{~T} N_{0}\right) \oplus \mathfrak{h}_{\mathcal{Z}} \oplus J\left(\mathfrak{h}_{\mathcal{Z}}\right) .
$$

Hence $J$ induces a compatible almost complex structure $J_{0}$ on $\left(N_{0}, \omega_{0}\right)$, such that $\left(\kappa_{J} \mid \mathcal{Z}\right) / H=\kappa_{J_{0}}$.

The line bundle $L_{2 \omega_{0}}=\left(L_{2 \omega} \mid \mathcal{Z}\right) / H$ is a prequantum line bundle on $\left(N_{0}, \omega_{0}\right)$. Finally, if we restrict $(2.6)$ to $\mathcal{Z}$, we get

$$
\tilde{\mathcal{L}}_{0}^{2}=L_{2 \omega_{0}} \otimes \kappa J_{0} .
$$

after taking the quotient by $H$. We have proved that $\left(J_{0}, \tilde{\mathcal{L}}_{0}\right)$ Spinprequantizes $\left(N_{0}, \omega_{0}\right)$.

For the rest of this section we consider a compact Hamiltonian $K$-manifold $(M, \omega, \Phi)$, that we suppose Spin-prequantized by the line bundle $\tilde{L}$.

Let $\tau$ be a face of the Weyl chamber, and let $K_{\tau}$ be the common stabilizer of points in $\tau$. Following Guillemin-Sternberg [13], we introduce the following $K_{\tau}$-invariant open subset of $\mathfrak{k}_{\tau}^{*}$ :

$$
U_{\tau}=K_{\tau} \cdot\left\{\xi \in \mathfrak{t}_{+}^{*} \mid K_{\xi} \subset K_{\tau}\right\}=K_{\tau} \cdot \bigcup_{\tau \subset \bar{\sigma}} \sigma .
$$

By construction, $U_{\tau}$ is a slice for the coadjoint action: this mean that the map $K \times U_{\tau},(k, \xi) \mapsto k \cdot \xi$ factors through an inclusion $K \times_{K_{\tau}} U_{\tau} \hookrightarrow \mathfrak{k}^{*}$.

The symplectic cross-section theorem [13] asserts that the pre-image $Y_{\tau}=$ $\Phi^{-1}\left(U_{\tau}\right)$ is a symplectic submanifold : we denote $\omega_{\tau}$ the restriction of $\omega$ to $Y_{\tau}$. The action of $K_{\tau}$ on $\left(Y_{\tau}, \omega_{\tau}\right)$ is Hamiltonian, where the restriction of $\Phi$ 
to $Y_{\tau}$ is a moment map. Since $\rho-\rho_{\tau}$ is a $K_{\tau}$-invariant element, we can use the translated moment map $\Phi_{\tau}: Y_{\tau} \rightarrow \mathfrak{k}_{\tau}^{*}$ defined by

$$
\Phi_{\tau}=\left.\Phi\right|_{Y_{\tau}}-\left(\rho-\rho_{\tau}\right) \text {. }
$$

Lemma 2.8. The symplectic slice $\left(Y_{\tau}, \omega_{\tau}, \Phi_{\tau}\right)$ is Spin-prequantized by the line bundle $\tilde{L}_{\tau}:=\left.\tilde{L}\right|_{Y_{\tau}}$.

Proof. We consider the open subset $K \times_{K_{\tau}} Y_{\tau}$ of $M$ and the projection $\pi: K \times_{K_{\tau}} Y_{\tau} \rightarrow K / K_{\tau}$. We can suppose that the Spin-prequantum data, when restricted to $K \times_{K_{\tau}} Y_{\tau}$, is given by $(J, \tilde{L})$ where $J$ is a compatible almost complex structure on $K \times_{K_{\tau}} Y_{\tau}$ defined as the "sum" of the compatible almost complex structures $J_{o}$ and $J_{\tau}: J_{o}$ on $K / K_{\tau}$ and $J_{\tau}$ on $Y_{\tau}$. Hence on $K \times K_{\tau} Y_{\tau}$ we have

$$
\kappa_{J}=K \times_{K_{\tau}}\left(\kappa_{J_{\tau}}\right) \otimes \pi^{-1}\left(\kappa_{J_{o}}\right)
$$

with $\kappa_{J_{o}}=K \times_{K_{\tau}} \mathbb{C}_{-2\left(\rho-\rho_{\tau}\right)}$. We see then that the restriction of the $K$ equivariant line bundle $\kappa_{J}$ to the symplectic slice $Y_{\tau}$ is equal to the $K_{\tau^{-}}$ equivariant line bundle $\kappa_{J_{\tau}} \otimes \mathbb{C}_{-2\left(\rho-\rho_{\tau}\right)}$.

When we restrict the identity $L_{2 \omega}=\tilde{L}^{2} \otimes \kappa_{J}^{-1}$ to $Y_{\tau}$ we get

$$
\left.L_{2 \omega}\right|_{Y_{\tau}}=\left(\left.\tilde{L}\right|_{Y_{\tau}}\right)^{2} \otimes \kappa_{J_{\tau}}^{-1} \otimes \mathbb{C}_{2\left(\rho-\rho_{\tau}\right)} .
$$

We consider the following line bundle on $Y_{\tau}$ :

$$
L_{2 \omega_{\tau}}:=\left.L_{2 \omega}\right|_{Y_{\tau}} \otimes \mathbb{C}_{-2\left(\rho-\rho_{\tau}\right)} .
$$

The relation (2.7) is then $L_{2 \omega_{\tau}}=\left(\left.\tilde{L}\right|_{Y_{\tau}}\right)^{2} \otimes \kappa_{J_{\tau}}^{-1}$. Since $L_{2 \omega_{\tau}}$ is a $K_{\tau^{-}}$ equivariant prequantum bundle over $\left(Y_{\tau}, 2 \omega_{\tau}, 2 \Phi_{\tau}\right)$, we conclude that $\left(Y_{\tau}, \omega_{\tau}\right.$, $\left.\Phi_{\tau}\right)$ is Spin-prequantized by the data $\left(J_{\tau},\left.\tilde{L}\right|_{Y_{\tau}}\right)$.

Let us consider the case where $\tau=\sigma$ is the smallest face of the Weyl chamber so that moment polyhedron $\Delta(M):=\Phi(M) \cap \mathfrak{t}_{+}^{*}$ is contained in the closure of $\sigma$.

Then the symplectic slice $Y_{\sigma}$ is equal to $\Phi^{-1}(\sigma)$, and the action of the subgroup $\left[K_{\sigma}, K_{\sigma}\right]$ is trivial on it $[\mathbf{1 7}]$. Let $Z_{\sigma}$ be the identity component of the center of $K_{\sigma}$. The map $\Phi_{\sigma}: Y_{\sigma} \rightarrow \mathfrak{k}_{\sigma}^{*}$ takes values in $\mathfrak{z}_{\sigma}^{*}=\mathbb{R} \sigma \subset \mathfrak{t}^{*}$ and corresponds to the moment map relative to the action of $Z_{\sigma}$ on $\left(Y_{\sigma}, \omega_{\sigma}\right)$. We know after Lemma 2.8 that $\left(Y_{\sigma}, \omega_{\sigma}, \Phi_{\sigma}\right)$ is Spin-prequantized by $\tilde{L}_{\sigma}:=\left.\tilde{L}\right|_{Y_{\sigma}}$.

For each dominants weights $\mu$ which belongs to the closure of $\sigma$, we consider the symplectic reduction

$$
\begin{aligned}
M_{\mu}^{\sigma} & =\Phi^{-1}\left(\mathcal{O}_{\mu}^{\sigma}\right) / K \\
& =\Phi_{\sigma}^{-1}(\mu) / Z_{\sigma} .
\end{aligned}
$$

For the rest of this section we fix a dominant weight $\mu \in \bar{\sigma}$ such that $\mu+\rho-\rho_{\sigma} \in \Delta(M)$, and we explain how one defines the Spin-quantization of the (possibly singular) reduced spaces $M_{\mu}^{\sigma}$. 
Let $\vec{\Delta} \subset \mathfrak{z}_{\sigma}^{*}$ be the rational vector subspace generated by $\{a-b \mid a, b \in$ $\Delta(M)\}$. Let $\mathfrak{z}_{\sigma}^{\Delta} \subset \mathfrak{z} \sigma$ be the subspace orthogonal to $\vec{\Delta}$, and let $Z_{\sigma}^{\Delta} \subset Z_{\sigma}$ be the corresponding subtorus.

Lemma 2.9. The torus $Z_{\sigma}^{\Delta}$ acts trivially on $Y_{\sigma}$ and on the line bundle $\tilde{L}_{\sigma} \otimes \mathbb{C}_{-\mu}$.

Proof. By definition of $\mathfrak{z}_{\sigma}^{\Delta}, 0=d\left\langle\Phi_{\sigma}, X\right\rangle=-\iota\left(X_{Y_{\sigma}}\right) \omega_{\sigma}$ on $Y_{\sigma}$ for any $X \in$ $\mathfrak{z}_{\sigma}^{\Delta}$. Hence the torus $Z_{\sigma}^{\Delta}$ acts trivially on $Y_{\sigma}$. Let $L_{2 \omega_{\sigma}}$ be the Kostant-Souriau line bundle over $\left(Y_{\sigma}, 2 \omega_{\sigma}, 2 \Phi_{\sigma}\right)$ so that $\tilde{L}_{\sigma}^{2}=L_{2 \omega_{\sigma}} \otimes \kappa_{J_{\sigma}}$ (see Lemma 2.8). We have on the section of $L_{2 \omega_{\sigma}}$ the following equality of linear operators:

$$
\mathcal{L}(X)-\nabla_{X_{M}}=\mathrm{i}\left\langle 2 \Phi_{\sigma}, X\right\rangle, \quad \forall X \in \mathfrak{z} \sigma .
$$

If one takes $X \in \mathfrak{z}_{\sigma}^{\Delta}$, the function $y \in Y_{\sigma} \mapsto\left\langle\Phi_{\sigma}(y), X\right\rangle$ is constant equal to $\langle\mu, X\rangle$. Finally

$$
\mathcal{L}(X)-2 \mathrm{i}\langle\mu, X\rangle=0, \quad \forall X \in \mathfrak{z}_{\sigma}^{\Delta}
$$

as an operator on the section of $L_{2 \omega_{\sigma}}$. In other words, the torus $Z_{\sigma}^{\Delta}$ acts trivially on $L_{2 \omega_{\sigma}} \otimes \mathbb{C}_{-2 \mu}=\left(\tilde{L}_{\sigma} \otimes \mathbb{C}_{-\mu}\right)^{2} \otimes \kappa_{J_{\sigma}}^{-1}$. Since $Z_{\sigma}^{\Delta}$ acts trivially on $\kappa_{J_{\sigma}}$, we conclude finally that $Z_{\sigma}^{\Delta}$ acts trivially on the line bundle $\tilde{L}_{\sigma} \otimes \mathbb{C}_{-\mu}$.

Let $Z_{\sigma}^{\prime} \subset Z_{\sigma}^{\Delta}$ be another subtorus such that $Z_{\sigma}=Z_{\sigma}^{\Delta} \times Z_{\sigma}^{\prime}$ : the dual of its Lie algebra $\mathfrak{z}_{\sigma}^{\prime}$ is identified with $\vec{\Delta} \subset \mathfrak{z}_{\sigma}^{*}$. We look now at $\left(Y_{\sigma}, \omega_{\sigma}\right)$ as a Hamiltonian $Z_{\sigma}^{\prime}$-manifold with moment map

$$
\Phi_{\sigma}^{\prime}:=\Phi_{\sigma}-\mu=\left.\Phi\right|_{Y_{\sigma}}-\left(\mu+\rho-\rho_{\sigma}\right) .
$$

The $Z_{\sigma}^{\prime}$-equivariant line bundle $\tilde{L}_{\sigma}^{\prime}:=\tilde{L}_{\sigma} \otimes \mathbb{C}_{-\mu}$ Spin-prequantizes the Hamiltonian $Z_{\sigma}^{\prime}$-manifold $\left(Y_{\sigma}, \omega_{\sigma}, \Phi_{\sigma}^{\prime}\right)$.

If $0 \in \vec{\Delta}$ is a regular value of $\Phi_{\sigma}^{\prime}$, we know after Lemma 2.7 that the orbifold reduced space $\left(M_{\mu}^{\sigma}, \omega_{\mu}^{\sigma}\right)$ is Spin-prequantized by the line bundle

$$
\tilde{\mathcal{L}}_{\mu}^{\sigma}:=\left(\left.\tilde{L}\right|_{\Phi_{\sigma}^{-1}(\mu)} \otimes \mathbb{C}_{-\mu}\right) / Z_{\sigma}^{\prime}
$$

and its Spin-quantization $\mathcal{Q}_{\text {spin }}\left(M_{\mu}^{\sigma}\right)$ is defined like in Definition 2.4. In the general case where $0 \in \vec{\Delta}$ is not necessarily a regular value of $\Phi_{\sigma}^{\prime}$ we proceed by shift desingularization. For $\varepsilon \in \vec{\Delta}$ small enough and generic we consider the orbifold reduced space

$$
M_{\mu+\varepsilon}^{\sigma}:=\left(\Phi_{\sigma}^{\prime}\right)^{-1}(\varepsilon) / Z_{\sigma}^{\prime}=\Phi_{\sigma}^{-1}(\mu+\varepsilon) / Z_{\sigma}^{\prime}
$$

and its orbifold line bundle

$$
\tilde{\mathcal{L}}_{\mu+\varepsilon}^{\sigma}:=\left(\left.\tilde{L}\right|_{\Phi_{\sigma}^{-1}(\mu+\varepsilon)} \otimes \mathbb{C}_{-\mu}\right) / Z_{\sigma}^{\prime} .
$$

The following crucial fact is proved in Section 3.4. 
Theorem 2.10. The Riemann-Roch number $R R\left(M_{\mu+\varepsilon}^{\sigma}, \tilde{\mathcal{L}}_{\mu+\varepsilon}^{\sigma}\right) \in \mathbb{Z}$ does not depend of the choice of a generic and small enough $\varepsilon \in \vec{\Delta}$.

Thanks to the last Theorem we can define the quantization $\mathcal{Q}_{\text {spin }}\left(M_{\mu}^{\sigma}\right) \in \mathbb{Z}$ of the (possibly singular) reduced space $M_{\mu}^{\sigma}$ for $\mu \in \widehat{K} \cap \bar{\sigma}$.

Definition 2.11. Let $\mu \in \widehat{K} \cap \bar{\sigma}$.

- If $\mu+\rho-\rho_{\sigma} \in \Delta(M)$, the integer $\mathcal{Q}_{\text {spin }}\left(M_{\mu}^{\sigma}\right) \in \mathbb{Z}$ is defined as the Riemann-Roch character $R R\left(M_{\mu+\varepsilon}^{\sigma}, \tilde{\mathcal{L}}_{\mu+\varepsilon}^{\sigma}\right)$ for $\varepsilon \in \vec{\Delta}$ generic and small enough.

- If $\mu+\rho-\rho_{\sigma} \notin \Delta(M)$, we set $\mathcal{Q}_{\text {spin }}\left(M_{\mu}^{\sigma}\right)=0$

Remark 2.12. If $\mu+\rho-\rho_{\sigma}$ does not belongs to the relative interior of $\Delta(M)$, we can choose $\varepsilon$ so that $\mu+\rho-\rho_{\sigma}+\varepsilon \notin \Delta(M)$. Then the reduced space $M_{\mu+\varepsilon}^{\sigma}$ is empty and the corresponding Riemann-Roch character $R R\left(M_{\mu+\varepsilon}^{\sigma}, \tilde{\mathcal{L}}_{\mu+\varepsilon}^{\sigma}\right)$ vanishes. Hence $\mathcal{Q}_{\text {spin }}\left(M_{\mu}^{\sigma}\right)=0$.

\section{Spin-quantization commutes with reduction}

Let $(M, \omega, \Phi)$ be a compact Hamiltonian $K$-manifold which is Spin prequantized. We are looking to a geometric interpretation of the multiplicity, denoted $\mathrm{m}_{\mu}$, of the representation $V_{\mu}^{K}$ into $\mathcal{Q}_{\text {spin }}^{K}(M)$.

The main result of this paper is the following.

Theorem 3.1. Let $\sigma$ be the smallest face of the Weyl chamber so that $\Phi(M) \cap \mathfrak{t}_{+}^{*} \subset \bar{\sigma}$. For $\mu \in \widehat{K}$, we have

$$
\mathrm{m}_{\mu}= \begin{cases}0 & \text { if } \mu \notin \bar{\sigma} ; \\ \mathcal{Q}_{\text {spin }}\left(M_{\mu}^{\sigma}\right) & \text { if } \mu \in \bar{\sigma} .\end{cases}
$$

In this section, we introduce the main tools needed for the proof of Theorem 3.1.

In Section 3.1, we recall the notion of transversally elliptic symbols.

In Section 3.2, we recall the Witten's way of localization the RiemannRoch character [21]. We recall in Proposition 3.8, the criterium observed in [22] for the vanishing of the invariant part of the localized RiemannRoch character.

In Section 3.3, we recall an induction formula proved in $[\mathbf{2 1}, \mathbf{2 2}]$ for the localized Riemann-Roch character.

In Section 3.4, we prove Theorem 3.1 when $K$ is a torus ${ }^{1}$. We give by the same way a proof of Theorem 2.10 which is essential to the definition of the Spin-quantization of the (possibly singular) reduced spaces $M_{\mu}^{\sigma}$.

\footnotetext{
${ }^{1}$ This situation was already handled in $[\mathbf{2 2}]$.
} 
3.1. Elliptic and transversally elliptic symbols. Here we give the basic definitions from the theory of transversally elliptic symbols (or operators) defined by Atiyah-Singer in [1]. For an axiomatic treatment of the index morphism see Berline-Vergne $[\mathbf{7 , 8}]$ and Paradan-Vergne [23]. For a short introduction see $[\mathbf{2 1}]$.

Let $\mathcal{X}$ be a compact $K$-manifold. Let $p: \mathrm{T} \mathcal{X} \rightarrow \mathcal{X}$ be the projection, and let $(-,-) \mathcal{X}$ be a $K$-invariant Riemannian metric. If $E^{0}, E^{1}$ are $K$-equivariant complex vector bundles over $\mathcal{X}$, a $K$-equivariant morphism $h \in \Gamma\left(\mathrm{T} \mathcal{X}, \operatorname{hom}\left(p^{*} E^{0}, p^{*} E^{1}\right)\right)$ is called a symbol on $\mathcal{X}$. The subset of all $(x, v) \in \mathrm{T} \mathcal{X}$ where $^{2} h(x, v): E_{x}^{0} \rightarrow E_{x}^{1}$ is not invertible is called the characteristic set of $h$, and is denoted by $\operatorname{Char}(h)$.

In the following, the "product" of a symbol $h$ by a complex vector bundle $F \rightarrow \mathcal{X}$, is the symbol

$$
h \otimes F
$$

defined by $h \otimes F(x, v)=h(x, v) \otimes \operatorname{Id}_{F_{x}}$ from $E_{x}^{0} \otimes F_{x}$ to $E_{x}^{1} \otimes F_{x}$. Note that $\operatorname{Char}(h \otimes F)=\operatorname{Char}(h)$.

Let $\mathrm{T}_{K} \mathcal{X}$ be the following subset of $\mathrm{T} \mathcal{X}$ :

$$
\mathrm{T}_{K} \mathcal{X}=\left\{(x, v) \in \mathrm{T} \mathcal{X},\left(v, X_{\mathcal{X}}(x)\right)_{\mathcal{X}}=0, \quad \text { for all } X \in \mathfrak{k}\right\} .
$$

A symbol $h$ is elliptic if $h$ is invertible outside a compact subset of T $\mathcal{X}$ (i.e., Char $(h)$ is compact), and is $K$-transversally elliptic if the restriction of $h$ to $\mathrm{T}_{K} \mathcal{X}$ is invertible outside a compact subset of $\mathrm{T}_{K} \mathcal{X}$ (i.e., $\operatorname{Char}(h) \cap \mathrm{T}_{K} \mathcal{X}$ is compact). An elliptic symbol $h$ defines an element in the equivariant $\mathbf{K}$ theory of $\mathrm{T} \mathcal{X}$ with compact support, which is denoted by $\mathbf{K}_{K}(\mathrm{~T} \mathcal{X})$, and the index of $h$ is a virtual finite-dimensional representation of $K$, that we denote $\operatorname{Index} K_{\mathcal{X}}^{K}(h) \in R(K)[\mathbf{2}-\mathbf{5}]$.

Let

$$
R_{\mathrm{tc}}^{-\infty}(K) \subset R^{-\infty}(K)
$$

be the $R(K)$-submodule formed by all the infinite sum $\sum_{\mu \in \widehat{K}} m_{\mu} V_{\mu}^{K}$ where the map $\mu \in \widehat{K} \mapsto m_{\mu} \in \mathbb{Z}$ has at most a polynomial growth. The $R(K)$ module $R_{\mathrm{tc}}^{-\infty}(K)$ is the Grothendieck group associated to the trace class virtual $K$-representations: we can associate to any $V \in R_{\mathrm{tc}}^{-\infty}(K)$, its trace $k \rightarrow \operatorname{Tr}(k, V)$ which is a generalized function on $K$ invariant by conjugation. Then the trace defines a morphism of $R(K)$-module

$$
R_{\mathrm{tc}}^{-\infty}(K) \hookrightarrow \mathcal{C}^{-\infty}(K)^{\mathrm{Ad}}
$$

where $\mathcal{C}^{-\infty}(K)^{\mathrm{Ad}}$ is the space of generalized function on $K$, which are invariant by conjugation.

A $K$-transversally elliptic symbol $h$ defines an element of $\mathbf{K}_{K}\left(\mathrm{~T}_{K} \mathcal{X}\right)$, and the index of $h$ is defined as a trace class virtual representation of $K$, that we still denote $\operatorname{Index}_{\mathcal{X}}^{K}(h) \in R_{\mathrm{tc}}^{-\infty}(K)$.

\footnotetext{
${ }^{2}$ The map $h(x, v)$ will be also denote $\left.h\right|_{x}(v)$
} 
Remark that any elliptic symbol of $\mathrm{T} \mathcal{X}$ is $K$-transversally elliptic, hence we have a restriction map $\mathbf{K}_{K}(\mathrm{~T} \mathcal{X}) \rightarrow \mathbf{K}_{K}\left(\mathrm{~T}_{K} \mathcal{X}\right)$, and a commutative diagram

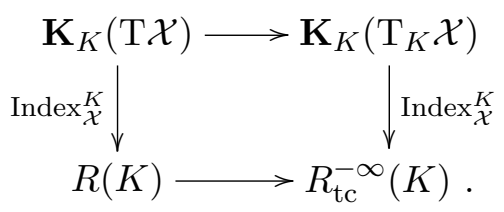

Using the excision property, one can easily show that the index map Index $\mathcal{U}_{\mathcal{U}}^{K}: \mathbf{K}_{K}\left(\mathrm{~T}_{K} \mathcal{U}\right) \rightarrow R_{\mathrm{tc}}^{-\infty}(K)$ is still defined when $\mathcal{U}$ is a $K$-invariant relatively compact open subset of a $K$-manifold (see [21, Section 3.1]).

Suppose that $M$ is a $K$-manifold equipped with an invariant almost complex structure $J$. Let us recall the definition of the Riemann-Roch character $R R_{J}^{K}(M,-)$.

The complex vector bundle $\left(\mathrm{T}^{*} M\right)^{0,1}$ is $K$-equivariantly identified with the tangent bundle TM equipped with the complex structure $J$. We work with the Hermitian structure on $(\mathrm{T} M, J)$ defined by $:(v, w):=\Omega(v, J w)-$ $\mathrm{i} \Omega(v, w)$ for $v, w \in \mathrm{T} M$. The symbol

$$
\operatorname{Thom}(M, J) \in \Gamma\left(\mathrm{T} M, \operatorname{hom}\left(p^{*}\left(\wedge_{\mathbb{C}}^{\text {even }} \mathrm{T} M\right), p^{*}\left(\wedge_{\mathbb{C}}^{\text {odd }} \mathrm{T} M\right)\right)\right)
$$

at $(m, v) \in \mathrm{T} M$ is equal to the Clifford map

$$
\mathbf{c}_{m}(v): \wedge_{\mathbb{C}}^{\text {even }} \mathrm{T}_{m} M \longrightarrow \wedge_{\mathbb{C}}^{\text {odd }} \mathrm{T}_{m} M,
$$

where $\mathbf{c}_{m}(v) . w=v \wedge w-\iota(v) w$ for $w \in \wedge_{\mathbb{C}}^{\bullet} \mathrm{T}_{m} M$. Here $\iota(v): \wedge_{\mathbb{C}}^{\bullet} \mathrm{T}_{m} M \rightarrow$ $\wedge_{\mathbb{C}}^{\bullet-1} \mathrm{~T}_{m} M$ denotes the contraction map. Since $\mathbf{c}_{m}(v)^{2}=-\|v\|^{2} \mathrm{Id}$, the map $\mathbf{c}_{m}(v)$ is invertible for all $v \neq 0$. Hence the characteristic set of $\operatorname{Thom}(M, J)$ corresponds to the 0 -section of TM.

Let $E$ be a $K$-equivariant complex vector bundle over $M$. It is a classical fact that the principal symbol of the Dolbeault-Dirac operator $\sqrt{2}\left(\bar{\partial}_{E}+\bar{\partial}_{E}^{*}\right)$ is equal to the following elliptic symbol ${ }^{3}$

$$
\mathbf{c}_{E}:=\operatorname{Thom}(M, J) \otimes E,
$$

see [6, Prop. 3.67]. Since $M$ is compact, the symbol $\mathbf{c}_{E}$ is elliptic and then defines an element of the equivariant $\mathbf{K}$-group of T $M$.

Definition 3.2. The Riemann-Roch character $R R_{J}^{K}(M, E) \in R(K)$ is defined equivalently

- as the topological index of $\mathbf{c}_{E} \in \mathbf{K}_{K}(\mathrm{~T} M)$, or

- as the analytical index of the Dolbeault-Dirac operator $\sqrt{2}\left(\bar{\partial}_{E}+\bar{\partial}_{E}^{*}\right)$.

\footnotetext{
${ }^{3}$ Here we use an identification $\mathrm{T}^{*} M \simeq \mathrm{T} M$ given by an invariant Riemannian metric.
} 
3.2. Localization of the Riemann-Roch character. Let $(M, \omega, \Phi)$ a compact Hamiltonian $K$-manifold Spin-prequantized by $(\tilde{L}, J)$ where $J$ is a compatible almost complex structure on $M$. The Riemann-Roch character attached to $J$ is just denoted $R R^{K}(M,-)$.

By definition the Spin-quantization of $(M, \omega, \Phi)$ is

$$
\mathcal{Q}_{\text {spin }}^{K}(M):=R R^{K}(M, \tilde{L}) \in R(K) .
$$

We recall the Witten's deformation of the Riemann-Roch character $[\mathbf{2 1}$, 22]. We use in all this paper an isomorphism $\mathfrak{k}^{*} \simeq \mathfrak{k}$ defined by a $K$-invariant scalar product on $\mathfrak{k}^{*}$. In order to simplify the notation, we use the same symbol for $\xi \in \mathfrak{k}^{*}$ and its corresponding element in $\mathfrak{k}$.

The moment map $\Phi$ is seen as en equivariant map from $M$ to $\mathfrak{k}$. We define the Kirwan vector field on $M$ :

$$
\kappa_{m}=(\Phi(m))_{M}(m), \quad m \in M .
$$

Definition 3.3. The symbol $\mathbf{c}_{\tilde{L}}=\operatorname{Thom}(M, J) \otimes \tilde{L}$ pushed by the vector field $\kappa$ is the symbol $\mathbf{c}_{\tilde{L}}^{\kappa}$ defined by the relation

$$
\left.\mathbf{c}_{\tilde{L}}^{\kappa}\right|_{m}(v)=\left.\operatorname{Thom}(M, J) \otimes \tilde{L}\right|_{m}\left(v-\kappa_{m}\right)
$$

for any $(m, v) \in \mathrm{T} M$.

Note that $\left.\mathbf{c}_{\tilde{L}}^{\kappa}\right|_{m}(v)$ is invertible except if $v=\kappa_{m}$. If furthermore $v$ belongs to the subset $\mathrm{T}_{K} M$ of tangent vectors orthogonal to the $K$-orbits, then $v=0$ and $\kappa_{m}=0$. Indeed $\kappa_{m}$ is tangent to $K \cdot m$ while $v$ is orthogonal. So we note that $(m, v) \in \operatorname{Char}\left(\mathbf{c}_{\tilde{L}}^{\kappa}\right) \cap \mathrm{T}_{K} M$ if and only if $v=0$ and $\kappa_{m}=0$.

Since $\kappa$ is the Hamiltonian vector field of the function $\frac{-1}{2}\|\Phi\|^{2}$, the set of zeros of $\kappa$ coincides with the set $\operatorname{Cr}\left(\|\Phi\|^{2}\right)$ of critical points of $\|\Phi\|^{2}$. Finally we have

$$
\begin{aligned}
\operatorname{Char}\left(\mathbf{c}_{\tilde{L}}^{\kappa}\right) \cap \mathrm{T}_{K} M & \simeq \operatorname{Cr}\left(\|\Phi\|^{2}\right) \\
& =\bigcup_{\beta \in \mathcal{B}} \underbrace{K \cdot\left(M^{\beta} \cap \Phi^{-1}(\beta)\right)}_{C_{\beta}},
\end{aligned}
$$

where $\mathcal{B}$ is the subset of the Weyl chamber defined by the relation $\beta \in \mathcal{B} \Longleftrightarrow$ $M^{\beta} \cap \Phi^{-1}(\beta) \neq \emptyset$. Recall the well-known fact.

Lemma 3.4. The set $\mathcal{B}$ is finite.

Proof. Let $\Phi_{T}: M \rightarrow \mathfrak{t}^{*}$ be the Hamiltonian action of a maximal torus of $K$. We have similarly $\operatorname{Cr}\left(\left\|\Phi_{T}\right\|^{2}\right)=\cup_{\beta \in \mathcal{B}_{T}} M^{\beta} \cap \Phi_{T}^{-1}(\beta)$, with $\mathcal{B}_{T} \subset \mathfrak{t}^{*}$. We see that $\mathcal{B} \subset \mathcal{B}_{T}$, hence it is sufficient to prove that $\mathcal{B}_{T}$ is finite. Let us consider the collection $\mathcal{B}^{\prime}$ of polytopes of $\mathfrak{t}^{*}$ which arise as the image by $\Phi_{T}$ of a connected component of the fixed point set $M^{H}$, where $H$ is a subtorus of $T$. It is easy to see that $\mathcal{B}^{\prime}$ is finite, and we checks in [20, Section 6.1] 
that any $\beta \in \mathcal{B}_{T}$ is equal to the orthogonal projection of $0 \in \mathfrak{t}^{*}$ to the affine space generated by some $\Delta \in \mathcal{B}^{\prime}$. Hence $\sharp \mathcal{B}_{T} \leq \sharp \mathcal{B}^{\prime}$ is finite.

We are interested to the restriction $\left.\mathbf{c}_{\tilde{L}}^{\kappa}\right|_{U}$ of the elliptic symbol on an invariant open subset $U \subset M$. Note that the set $\operatorname{Char}\left(\left.\mathbf{c}_{\tilde{L}}^{\kappa}\right|_{U}\right) \cap \mathrm{T}_{K} U \simeq$ $\operatorname{Cr}\left(\|\Phi\|^{2}\right) \cap U$ is compact when

$$
\partial \mathcal{U} \cap \operatorname{Cr}\left(\|\Phi\|^{2}\right)=\emptyset .
$$

When (3.5) holds we denote

$$
\mathcal{Q}_{\Phi}^{K}(U):=\operatorname{Index}_{U}^{K}\left(\left.\mathbf{c}_{\tilde{L}}^{\kappa}\right|_{U}\right) \quad \in \quad R_{\mathrm{tc}}^{-\infty}(K)
$$

the equivariant index of the transversally elliptic symbol $\left.\mathbf{c}_{\tilde{L}}^{\kappa}\right|_{U}$.

For any $\beta \in \mathcal{B}$, we consider a relatively compact open invariant neighborhood $U_{\beta}$ of $C_{\beta}$ such that $\operatorname{Cr}\left(\|\Phi\|^{2}\right) \cap \overline{U_{\beta}}=C_{\beta}$.

Definition 3.5. We denote

$$
\mathcal{Q}_{\beta}^{K}(M) \in R_{\mathrm{tc}}^{-\infty}(K)
$$

the index of the transversally elliptic symbol $\left.\mathbf{c}_{\tilde{L}}^{\kappa}\right|_{U_{\beta}}$.

Everything can be defined if we replace the line bundle $\tilde{L}$ by any equivariant complex vector bundle $E$. We can consider the pushed symbol $\mathbf{c}_{E}^{\kappa}$, and the localized Riemann-Roch characters

$$
R R_{\Phi}^{K}(U, E):=\operatorname{Index}_{U}^{K}\left(\left.\mathbf{c}_{E}^{\kappa}\right|_{U}\right) \text { and } R R_{\beta}^{K}(M, E):=\operatorname{Index}_{U_{\beta}}^{K}\left(\left.\mathbf{c}_{E}^{\kappa}\right|_{U_{\beta}}\right) .
$$

A direct application of the excision property [21, Section 4] gives that

$$
\mathcal{Q}_{\text {spin }}^{K}(M)=\sum_{\beta \in \mathcal{B}} \mathcal{Q}_{\beta}^{K}(M) .
$$

If we work with $R R_{\Phi}^{K}(U, E)$, we have

$$
R R_{\Phi}^{K}(U, E)=\sum_{\beta \in \mathcal{B} \cap \Phi(U)} R R_{\beta}^{K}(U, E) .
$$

The decomposition (3.7) and (3.8) will be used in the next chapters when one want to compute the multiplicity, denoted $\left[\mathcal{Q}_{\text {spin }}^{K}(M)\right]^{K}$, of the trivial representation in $\mathcal{Q}_{\text {spin }}^{K}(M)$. We have

$$
\left[\mathcal{Q}_{\text {spin }}^{K}(M)\right]^{K}=\sum_{\beta \in \mathcal{B}}\left[\mathcal{Q}_{\beta}^{K}(M)\right]^{K} .
$$

and we finish this section by recalling a criterium under which one has $\left[\mathcal{Q}_{\beta}^{K}(M)\right]^{K}=0$.

Let $\beta$ be a non-zero element in $\mathfrak{k}$ : let $\mathbb{T}_{\beta} \subset K$ be the torus generated by $\beta$. For $m \in M^{\beta}$, let $\alpha_{1}^{m}, \ldots, \alpha_{p}^{m}$ be the real infinitesimal weights for the action of $\mathbb{T}_{\beta}$ on the fibers of $\mathrm{T}_{m} M$ (we equip the fibers of $\mathrm{T}_{m} M / \mathrm{T}_{m} M^{\beta}$ with a $\mathbb{T}_{\beta}$-invariant complex structure). 
Definition 3.6. Let us denote by $\operatorname{Tr}_{\beta}\left|\mathrm{T}_{m} M\right|$ the following positive number

$$
\operatorname{Tr}_{\beta}\left|\mathrm{T}_{m} M\right|:=\sum_{i=1}^{l}\left|\left\langle\alpha_{i}^{m}, \beta\right\rangle\right| .
$$

Note that $m \in M^{\beta} \mapsto \operatorname{Tr}_{\beta}\left|\mathrm{T}_{m} M\right|$ is constant along a connected component of $M^{\beta}$. We see also that the expression $\operatorname{Tr}_{\beta}|E|$ is well defined for any $H$-equivariant real vector bundle $E \rightarrow P$, when $\beta \in \mathfrak{h}$ acts trivially on $P$.

Example 3.7. The map $\beta \in \mathfrak{k} \mapsto \operatorname{Tr}_{\beta}|\mathfrak{k}|$ is invariant under the adjoint action. When $\beta$ belongs to the Weyl chamber, one has $\operatorname{Tr}_{\beta}|\mathfrak{k}|=2(\rho, \beta)$. Note that $\operatorname{Tr}_{\beta}|\mathfrak{k}| \leq 2\|\rho\|\|\beta\|$ for any $\beta \in \mathfrak{k}$.

We have proved in [22, Proposition 3.11] the following useful criterium.

Proposition 3.8. Let $\beta \neq 0$ in $\mathcal{B}$. The multiplicity of the trivial representation in $\mathcal{Q}_{\beta}^{K}(M)$ is equal to zero if

$$
\|\beta\|^{2}+\frac{1}{2} \boldsymbol{T r}_{\beta}\left|\mathrm{T}_{m} M\right|>\boldsymbol{T r}_{\beta}|\mathfrak{k}|, \quad \forall m \in M^{\beta} \cap \Phi^{-1}(\beta) .
$$

Remark 3.9. Note that condition (3.9) is equivalent to

$$
\|\Phi(m)\|^{2}+\frac{1}{2} \operatorname{Tr}_{\Phi(m)}\left|\mathrm{T}_{m} M\right|>\operatorname{Tr}_{\Phi(m)}|\mathfrak{k}|, \quad \forall m \in C_{\beta} .
$$

If the critical set $C_{\beta}$ decomposes in a finite disjoint union of closed $K$ invariant subset $C_{\beta}=\cup_{j} C_{\beta}^{j}$, we consider invariant open neighborhood $U^{j}$ of $C_{\beta}^{j}$ such that $\overline{U_{\beta}^{j}} \cap \operatorname{Cr}\left(\|\Phi\|^{2}\right)=C_{\beta}^{j}$, and we define

$$
\mathcal{Q}_{C_{\beta}^{j}}^{K}(M):=\operatorname{Index}_{U_{\beta}^{j}}^{K}\left(\left.\mathbf{c}^{\kappa}\right|_{U_{\beta}^{j}}\right) \quad \in \quad R_{\mathrm{tc}}^{-\infty}(K) .
$$

Then the generalized character $\mathcal{Q}_{\beta}^{K}(M)$ is equal to the sum $\sum_{j} \mathcal{Q}_{C_{\beta}^{j}}^{K}(M)$ and Proposition 3.8 tells us that $\left[\mathcal{Q}_{C_{\beta}^{j}}^{K}(M)\right]^{K}=0$ if (3.10) holds on $C_{\beta}^{j}$.

3.3. Induction formulas. Let $H$ be a compact connected Lie group. Let $H \cdot a$ be a coadjoint orbit. Let $\left(N, \omega_{N}, \Phi_{N}\right)$ be an Hamiltonian $H$-manifold which is not assumed to be compact. But we assume that $\Phi_{N}$ is proper near $H \cdot a$ : the pullback $\Phi_{N}^{-1}(\mathcal{C})$ is compact if $\mathcal{C} \subset \mathfrak{h}^{*}$ is a small-enough compact invariant neighborhood of $H \cdot a$.

Let $H_{a}$ be the stabilizer of $a \in \mathfrak{h}^{*}$, and let $Y_{a}$ be a symplectic slice near $H \cdot a$ : $Y_{a}$ is a $H_{a}$-invariant symplectic manifold of $N$ such that $\Phi_{N}\left(Y_{a}\right) \subset \mathfrak{h}_{a}^{*}$ and such that $H \times{ }_{H_{a}} Y_{a}$ is diffeomorphic to an invariant open neighborhood of $\Phi_{N}^{-1}(H \cdot a)$. We will work with the following moment map on $Y_{a}$ :

$$
\Phi_{Y_{a}}=\left.\Phi_{N}\right|_{Y_{a}}-a .
$$

Let $N \times \overline{H \cdot a}$ be the Hamiltonian $H$-manifold, with moment map $\Phi(n, \xi)=\Phi_{N}(n)-\xi$. Let

$$
R R_{0}^{H}(N \times \overline{H \cdot a},-)
$$


be the Riemann-Roch character localized near the compact subset $\Phi^{-1}(0) \subset$ $N \times \overline{H \cdot a}$. Let

$$
R R_{0}^{H_{a}}\left(Y_{a},-\right)
$$

be the Riemann-Roch character localized near the compact subset $\Phi_{Y_{a}}^{-1}(0)=$ $\Phi_{N}^{-1}(a) \subset Y_{a}$.

Let $\operatorname{Ind}_{H_{a}}^{H}: R^{-\infty}\left(H_{a}\right) \rightarrow R^{-\infty}(H)$ be the induction map. If $E$ and $F$ are respectively $H$-equivariant complex vector bundles on $N$ and $H \cdot a$, we denote $E \otimes F$ their product. We have proved in [21, Proposition 7.10] (see also [22, Proposition 4.13]) the following induction formula.

Proposition 3.10. For any equivariant complex vector bundles $E \rightarrow N$ and $F \rightarrow H \cdot a$, we have

$$
R R_{0}^{H}(N \times \overline{H \cdot a}, E \otimes F)=\operatorname{Ind}_{H_{a}}^{H}\left[R R_{0}^{H_{a}}\left(Y_{a},\left.\left.E\right|_{Y_{a}} \otimes F\right|_{\{a\}}\right)\right] .
$$

The last Proposition gives in particular that

$$
\left[R R_{0}^{H}(N \times \overline{H \cdot a}, E \otimes F)\right]^{H}=\left[R R_{0}^{H_{a}}\left(Y_{a},\left.\left.E\right|_{Y_{a}} \otimes F\right|_{\{a\}}\right)\right]^{H_{a}} .
$$

3.4. The torus case. Let $T$ be a compact torus, and let $(M, \omega, \Phi)$ be a compact Hamiltonian $T$-manifold which is Spin-prequantized by the data $(J, \tilde{L})$. We suppose that $J$ is compatible with $\omega$. The irreducible representation of $T$ is parametrized by the lattice $\widehat{T} \subset \mathfrak{t}^{*}$ : at each $\mu \in \widehat{T}$ we associate the one-dimensional representation $\mathbb{C}_{\mu}$.

We write $\mathcal{Q}_{\text {spin }}^{T}(M)=\sum_{\mu \in \widehat{T}} \mathrm{~m}_{\mu} \mathbb{C}_{\mu}$, and one wants to show that the multiplicity $\mathrm{m}_{\mu}$ is equal to the Spin-quantization of the (possibly singular) reduced space $M_{\mu}:=\Phi^{-1}(\mu) / T$.

We fix once for all $\mu \in \widehat{T}$. And we apply the Witten deformation procedure to the Hamiltonian $T$-manifold $(M, \omega, \Phi-\mu)$ which is Spin-prequantized by $\left(J, \tilde{L} \otimes \mathbb{C}_{-\mu}\right)$. We have

$$
\mathrm{m}_{\mu}=\sum_{\beta \in \mathcal{B}^{\mu}}\left[R R_{\beta}^{T}\left(M, \tilde{L} \otimes \mathbb{C}_{-\mu}\right)\right]^{\mathrm{T}},
$$

where $\mathcal{B}^{\mu}$ parametrizes the critical points of $\|\Phi-\mu\|^{2}$. Here the criterion (3.9) holds for any non-zero $\beta$ since the Lie algebra $\mathfrak{t}$ is abelian. We have then

$$
\mathrm{m}_{\mu}=\left[R R_{0}^{T}\left(M, \tilde{L} \otimes \mathbb{C}_{-\mu}\right)\right]^{\mathrm{T}} .
$$

In particular, $\mathrm{m}_{\mu}=0$ if $\mu \notin \Phi(M)$. When $\mu \in \Phi(M)$, we consider a small neighborhood $U$ of $\Phi^{-1}(\mu) \subset M$ so that $\bar{U} \cap \operatorname{Cr}\left(\|\Phi-\mu\|^{2}\right)=\Phi^{-1}(\mu)$. We know then that

$$
\mathrm{m}_{\mu}=\left[R R_{\Phi-\mu}^{T}\left(U,\left.\tilde{L}\right|_{U} \otimes \mathbb{C}_{-\mu}\right)\right]^{\mathrm{T}}
$$


3.4.1. First case: $\boldsymbol{\mu}$ is a regular value of $\boldsymbol{\Phi}$. We consider the orbifold reduced space $M_{\mu}=\Phi^{-1}(\mu) / T$ which is equipped with a canonical symplectic form $\omega_{\mu}$. Let $R R\left(M_{\mu},-\right)$ be the Riemann-Roch character attached to a compatible almost complex structure. We prove in [21, Section 7.1] that for any complex vector bundle $E \rightarrow U$

$$
\left[R R_{\Phi-\mu}^{T}(U, E)\right]^{T}=R R\left(M_{\mu}, \mathcal{E}\right)
$$

where $\mathcal{E}=\left.E\right|_{\Phi^{-1}(\mu)} / T$ is the induced orbifold bundle on $M_{\mu}$. If we take $E=\left.\tilde{L}\right|_{U} \otimes \mathbb{C}_{-\mu}$ on sees (thanks to Lemma 2.7) that

$$
\tilde{\mathcal{L}}_{\mu}=\left(\left.\tilde{L}\right|_{\Phi^{-1}(\mu)} \otimes \mathbb{C}_{-\mu}\right) / T
$$

is an orbifold line bundle which Spin-prequantizes $\left(M_{\mu}, \omega_{\mu}\right)$, and (3.13) gives together with (3.12) that

$$
\mathrm{m}_{\mu}=R R\left(M_{\mu}, \tilde{\mathcal{L}}_{\mu}\right)=\mathcal{Q}_{\text {spin }}\left(M_{\mu}\right) .
$$

3.4.2. Second case: $\boldsymbol{\mu}$ is a not (necessarily) a regular value of $\Phi$. Let $\vec{\Delta} \subset \mathfrak{t}^{*}$ be the rational vector subspace generated by $\{a-b \mid a, b \in \Phi(M)\}$. We work here with a weight $\mu \in \Phi(M)$ so that the polytope $\Phi(M)$ lives in the affine subspace $\mu+\vec{\Delta}$. Let $\mathfrak{t}_{\Delta} \subset \mathfrak{t}$ be the subspace orthogonal to $\Delta$, and let $T_{\Delta} \subset T$ be the corresponding subtorus.

Lemma 3.11. The group $T_{\Delta}$ acts trivially on $M$ and on the line bundle $\tilde{L} \otimes \mathbb{C}_{-\mu}$.

Proof. See the proof of Lemma 2.9 .

Let $T^{\prime} \subset T$ be another subtorus such that $T=T_{\Delta} \times T^{\prime}$ : the dual of its Lie algebra $\mathfrak{t}^{\prime}$ is identified with $\vec{\Delta} \subset \mathfrak{t}^{*}$. We look now at $(M, \omega)$ as a Hamiltonian $T^{\prime}$-manifold with moment map

$$
\Phi^{\prime}:=\Phi-\mu: M \longrightarrow \vec{\Delta}=\left(\mathfrak{t}^{\prime}\right)^{*} .
$$

The $T^{\prime}$-equivariant line bundle $\tilde{L}^{\prime}:=\tilde{L} \otimes \mathbb{C}_{-\mu}$ Spin-prequantizes the Hamiltonian $T^{\prime}$-manifold $\left(M, \omega, \Phi^{\prime}\right)$. Let $U$ be a small neighborhood of $\Phi^{\prime-1}(0)$ in $M$. The generalized character $R R_{\Phi-\mu}^{T}\left(U,\left.\tilde{L}\right|_{U} \otimes \mathbb{C}_{-\mu}\right)$ belongs to $R^{-\infty}\left(T^{\prime}\right)$ and corresponds to the localized Riemann-Roch character

$$
R R_{\Phi^{\prime}}^{T^{\prime}}\left(U,\left.\tilde{L}^{\prime}\right|_{U}\right)
$$

We deform the moment map $\Phi^{\prime}$ in $\Phi^{\prime}-\varepsilon$ where $\varepsilon$ is a small element in $\vec{\Delta}$. We have proved in [22, Proposition 4.14] the following

Lemma 3.12. - If $\varepsilon$ is small enough, the critical set of $\left\|\Phi^{\prime}-\varepsilon\right\|^{2}$ does not intersect $\partial U$, so that the localized Riemann-Roch character $R R_{\Phi^{\prime}-\varepsilon}^{T^{\prime}}(U,-)$ is well defined.

- We have $R R_{\Phi^{\prime}}^{T^{\prime}}\left(U,\left.\tilde{L}^{\prime}\right|_{U}\right)=R R_{\Phi^{\prime}-\varepsilon}^{T^{\prime}}\left(U,\left.\tilde{L}^{\prime}\right|_{U}\right)$ if $\varepsilon$ is small enough. 
Now we are left to the computation of $\mathrm{m}_{\mu}=\left[R R_{\Phi^{\prime}-\varepsilon}^{T^{\prime}}\left(U,\left.\tilde{L}^{\prime}\right|_{U}\right)\right]^{T^{\prime}}$ when $\varepsilon \in \vec{\Delta}$ is small enough. We start with the decomposition

$$
R R_{\Phi^{\prime}-\varepsilon}^{T^{\prime}}\left(U,\left.\tilde{L}^{\prime}\right|_{U}\right)=\sum_{\beta \in \mathcal{B}_{\varepsilon}} R R_{\Phi^{\prime}-\varepsilon, \beta}^{T^{\prime}}\left(U,\left.\tilde{L}^{\prime}\right|_{U}\right)
$$

where $R R_{\Phi^{\prime}-\varepsilon, \beta}^{T^{\prime}}(U,-)$ denotes the Riemann-Roch character localized near the compact subset $U^{\beta} \cap\left(\Phi^{\prime}\right)^{-1}(\beta+\varepsilon)$. We have proved in [22, Lemma 4.16] the following

Lemma 3.13. If $\varepsilon$ is small enough we have $\left[R R_{\Phi^{\prime}-\varepsilon, \beta}^{T^{\prime}}\left(U,\left.\tilde{L}^{\prime}\right|_{U}\right)\right]^{T^{\prime}}=0$ when $\beta \neq 0$.

At $\varepsilon \in \vec{\Delta}$ small enough and generic we associate the orbifold $M_{\mu+\varepsilon}=$ $\Phi^{-1}(\mu+\varepsilon) / T^{\prime}$, which is equipped with the orbifold line bundle

$$
\tilde{\mathcal{L}}_{\mu+\varepsilon}=\left(\left.\tilde{L}\right|_{\Phi^{-1}(\mu+\varepsilon)} \otimes \mathbb{C}_{-\mu}\right) / T^{\prime} .
$$

Let $R R\left(M_{\mu+\varepsilon},-\right)$ be the Riemann-Roch map associated to a compatible almost complex structure. If we use (3.13) together with the Lemmas 3.12 and 3.13 we get:

Theorem 3.14. The multiplicity $\mathrm{m}_{\mu}$ is equal to the Riemann-Roch number $R R\left(M_{\mu+\varepsilon}, \tilde{\mathcal{L}}_{\mu+\varepsilon}\right) \in \mathbb{Z}$ where $\varepsilon \in \vec{\Delta}$ is small and generic.

We prove here that the quantity $R R\left(M_{\mu+\varepsilon}, \tilde{\mathcal{L}}_{\mu+\varepsilon}\right)$ does not depend of the choice of $\varepsilon$ small and generic: it is the definition of the Spin quantization, denoted $\mathcal{Q}_{\text {spin }}\left(M_{\mu}\right)$, of the (possibly singular) reduced space $M_{\mu}$.

3.4.3. Proof of Theorem 2.10. The same kind of proof work for Theorem 2.10. We consider an invariant relatively compact neighborhood $U_{\sigma, \mu}$ of $\Phi_{\sigma}^{-1}(\mu)=\Phi^{-1}\left(\mu+\rho-\rho_{\sigma}\right)$ in the slice $Y_{\sigma}$ so that $\operatorname{Cr}\left(\left\|\Phi_{\sigma}-\mu\right\|^{2}\right) \cap \overline{U_{\sigma, \mu}}=$ $\Phi_{\sigma}^{-1}(\mu)$. Thanks to Lemmas 3.12 and 3.13, we know that the Riemann-Roch character

$$
R R_{\Phi_{\sigma}-\mu-\varepsilon}^{Z_{\sigma}^{\prime}}\left(U_{\sigma, \mu}, \tilde{L}^{\prime}\right) \in R^{-\infty}\left(Z_{\sigma}^{\prime}\right)
$$

are well defined for $\varepsilon \in \vec{\Delta}$ small enough, and they do not depend of the choice of $\varepsilon$. If $\varepsilon_{1}, \varepsilon_{2} \in \vec{\Delta}$ are small enough regular values of $\Phi_{\sigma}-\mu$ we get thanks to (3.13) that

$$
\begin{aligned}
R R\left(M_{\mu+\varepsilon_{1}}^{\sigma}, \tilde{\mathcal{L}}_{\mu+\varepsilon_{1}}^{\sigma}\right) & =\left[R R_{\Phi_{\sigma}-\mu-\varepsilon_{1}}^{Z_{\sigma}^{\prime}}\left(U_{\sigma, \mu}, \tilde{L}^{\prime}\right)\right]^{Z_{\sigma}^{\prime}} \\
& =\left[R R_{\Phi_{\sigma}-\mu-\varepsilon_{2}}^{Z_{\sigma}^{\prime}}\left(U_{\sigma, \mu}, \tilde{L}^{\prime}\right)\right]^{Z_{\sigma}^{\prime}} \\
& =R R\left(M_{\mu+\varepsilon_{2}}^{\sigma}, \tilde{\mathcal{L}}_{\mu+\varepsilon_{2}}^{\sigma}\right) .
\end{aligned}
$$




\section{Proof of Theorem 3.1}

Let $(M, \omega, \Phi)$ be a compact Hamiltonian $K$-manifold which is Spin prequantized. Let $\sigma$ be the smallest face of the Weyl chamber so that $\Phi(M) \cap \mathfrak{t}_{+}^{*} \subset \bar{\sigma}$. Let $\mu$ be a dominant weight, and let $\mathrm{m}_{\mu}$ be the multiplicity of $V_{\mu}^{K}$ in $\mathcal{Q}_{\text {spin }}^{K}(M)$.

Let $\mathcal{O}_{\mu}$ be the coadjoint orbit $K \cdot(\mu+\rho)$. Since the dual representation $\left(V_{\mu}^{K}\right)^{*}$ can be realized ${ }^{4}$ as $\mathcal{Q}_{\text {spin }}^{K}\left(\overline{\mathcal{O}_{\mu}}\right)$, we know by the shifting trick that

$$
\mathrm{m}_{\mu}=\left[\mathcal{Q}_{\text {spin }}^{K}\left(M \times \overline{\mathcal{O}_{\mu}}\right)\right]^{K} .
$$

Now we work with the Hamiltonian $K$-manifold $N=M \times \overline{\mathcal{O}_{\mu}}$ with moment map $\Phi_{N}(m, \xi)=\Phi(m)-\xi$. The Witten's deformation on $N$ gives $\mathcal{Q}_{\text {spin }}^{K}\left(M \times \overline{\mathcal{O}_{\mu}}\right)=\sum_{\beta \in \mathcal{B}^{\mu}} \mathcal{Q}_{\beta}^{K}\left(M \times \overline{\mathcal{O}_{\mu}}\right)$ where $\mathcal{B}^{\mu}$ is a finite set parametrizing $\operatorname{Cr}\left(\left\|\Phi_{N}\right\|^{2}\right)$, and $\mathcal{Q}_{\beta}^{K}\left(M \times \overline{\mathcal{O}_{\mu}}\right)$ is an index of a transversally elliptic operator localized near $C_{\beta}=K\left(N^{\beta} \cap \Phi_{N}^{-1}(\beta)\right)$.

We have then

$$
\mathrm{m}_{\mu}=\sum_{\beta \in \mathcal{B}^{\mu}}\left[\mathcal{Q}_{\beta}^{K}\left(M \times \overline{\mathcal{O}_{\mu}}\right)\right]^{K}
$$

We remark that 0 does not appears in $\mathcal{B}^{\mu}$ when $\sigma \neq \mathfrak{t}_{+}^{*}$, since $\mu+\rho \notin \Phi(M)$.

The main point of this section is the following:

Proposition 4.1. - If $\mu \notin \bar{\sigma}$, the identity (3.10) holds on $C_{\beta}$ for any $\beta \in \mathcal{B}^{\mu}$. Hence $\mathrm{m}_{\mu}=0$.

- If $\mu \in \bar{\sigma}$, the identity (3.10) holds on $C_{\beta}$ for any $\beta \neq-\rho_{\sigma}$. Then

$$
\mathrm{m}_{\mu}=\left[\mathcal{Q}_{-\rho_{\sigma}}^{K}\left(M \times \overline{\mathcal{O}_{\mu}}\right)\right]^{K} .
$$

When $\sigma=\mathfrak{t}_{+}^{*}$, we have $\rho_{\sigma}=0$ and Proposition 4.1 tell us that the multiplicity $\mathrm{m}_{\mu}$ id equal to $\left[\mathcal{Q}_{0}^{K}\left(M \times \overline{\mathcal{O}_{\mu}}\right)\right]^{K}$ for any $\mu \in \widehat{K}$. In particular, $\mathrm{m}_{\mu}=0$ if $\mu+\rho \notin \Phi(M)$.

When $\sigma \neq \mathfrak{t}_{+}^{*}$ and $\mu \in \bar{\sigma}$, we precise Proposition 4.1 as follow. The generalized character $\mathcal{Q}_{-\rho_{\sigma}}^{K}\left(M \times \overline{\mathcal{O}_{\mu}}\right)$ is defined as the index of a transversally elliptic symbol living in a neighborhood of

$$
C_{-\rho_{\sigma}}=K\left(N^{\rho_{\sigma}} \cap \Phi_{N}^{-1}\left(-\rho_{\sigma}\right)\right) .
$$

Let $K_{\rho_{\sigma}}$ be the stabilizer subgroup of $\rho_{\sigma}$. Let $W\left(K_{\rho_{\sigma}}\right) \subset W$ be the Weyl subgroup of $K_{\rho_{\sigma}}$. A direct computation gives that

$$
C_{-\rho_{\sigma}}=\bigcup_{\bar{w} \in W\left(K_{\rho_{\sigma}}\right) \backslash W} C_{-\rho_{\sigma}, \bar{w}}
$$

\footnotetext{
${ }^{4} \overline{\mathcal{O}_{\mu}}$ is the coadjoint orbit $\mathcal{O}_{\mu}$ with the opposite symplectic structure.
} 
with $C_{-\rho_{\sigma}, \bar{w}}=K\left(M^{\rho_{\sigma}} \cap \Phi^{-1}\left(w(\mu+\rho)-\rho_{\sigma}\right) \times\{w(\mu+\rho)\}\right)$. We are particularly interested in the component ${ }^{5}$

$$
C_{-\rho_{\sigma}, \bar{e}}:=K\left(M^{\rho_{\sigma}} \cap \Phi^{-1}\left(\mu+\rho-\rho_{\sigma}\right) \times\{\mu+\rho\}\right) .
$$

Let us denote $C_{-\rho_{\sigma}, \text { out }}$ the union of the $C_{-\rho_{\sigma}, \bar{w}}$ for $\bar{w} \neq \bar{e}$. We have a decomposition

$$
C_{-\rho_{\sigma}}=C_{-\rho_{\sigma}, \bar{e}} \cup C_{-\rho_{\sigma}, \text { out }}
$$

into closed invariant disjoint subsets. Then the generalized character $\mathcal{Q}_{-\rho_{\sigma}}^{K}\left(M \times \overline{\mathcal{O}_{\mu}}\right)$ is equal to the sum

$$
\mathcal{Q}_{-\rho_{\sigma}, \bar{e}}^{K}\left(M \times \overline{\mathcal{O}_{\mu}}\right)+\mathcal{Q}_{-\rho_{\sigma}, \text { out }}^{K}\left(M \times \overline{\mathcal{O}_{\mu}}\right)
$$

where both terms correspond to the specialization of the transversally elliptic symbol to the neighborhood of each part of the decomposition (4.2).

Proposition 4.2. Suppose that $\sigma \neq \mathfrak{t}_{+}^{*}$ and that $\mu \in \bar{\sigma}$. The identity (3.10) holds on the subset $C_{-\rho_{\sigma}, \text { out }}$, and then

$$
\mathrm{m}_{\mu}=\left[\mathcal{Q}_{-\rho_{\sigma}, \bar{e}}^{K}\left(M \times \overline{\mathcal{O}_{\mu}}\right)\right]^{K} .
$$

Note that $C_{-\rho_{\sigma}, \bar{e}}=\emptyset$ if $\mu+\rho-\rho_{\sigma} \notin \Phi(M)$. At this stage we know then that $\mathrm{m}_{\mu}=0$ if $\mu+\rho-\rho_{\sigma}$ does not belongs to the image of the moment map.

4.1. Proofs of Propositions 4.1 and 4.2. Let $N=M \times \overline{\mathcal{O}_{\mu}}$ and let $\left\|\Phi_{N}\right\|^{2}: N \rightarrow \mathbb{R}$ be the square of the moment map. Recall that we denote by $\sigma$ the smallest face of the Weyl chamber so that $\Phi(M) \cap \mathbf{t}_{+}^{*} \subset \bar{\sigma}$.

We want to prove that for any $n=(m, \xi) \in \operatorname{Cr}\left(\left\|\Phi_{N}\right\|^{2}\right)$ the vector $\beta:=$ $\Phi(m)-\xi$ satisfies

$$
\|\beta\|^{2}+\frac{1}{2} \operatorname{Tr}_{\beta}\left|\mathrm{T}_{n} N\right| \geq \operatorname{Tr}_{\beta}|\mathfrak{k}|
$$

Afterwards we will discuss the case of equality in (I).

The tangent space $\mathrm{T}_{\xi} \mathcal{O}_{\mu}$ is equal to the $\mathfrak{k}_{\xi}$-module $\mathfrak{k} / \mathfrak{k}_{\xi}$ : then

$$
\begin{aligned}
\operatorname{Tr}_{\beta}\left|\mathrm{T}_{\xi} \mathcal{O}_{\mu}\right| & =\operatorname{Tr}_{\beta}|\mathfrak{k}|-\operatorname{Tr}_{\beta}\left|\mathfrak{k}_{\xi}\right| \\
& =\operatorname{Tr}_{\beta}|\mathfrak{k}|,
\end{aligned}
$$

since $\beta$ belongs to the abelian subalgebra $\mathfrak{k}_{\xi}$. Using that $\operatorname{Tr}_{\beta}\left|\mathrm{T}_{n} N\right|=$ $\operatorname{Tr}_{\beta}\left|\mathrm{T}_{m} M\right|+\operatorname{Tr}_{\beta}|\mathfrak{k}|$, we see that (I) is equivalent to

$$
\|\beta\|^{2}+\frac{1}{2} \operatorname{Tr}_{\beta}\left|\mathrm{T}_{m} M\right| \geq \frac{1}{2} \operatorname{Tr}_{\beta}|\mathfrak{k}| .
$$

The module $\mathfrak{k} / \mathfrak{k}_{m}$ is naturally a subspace of $\mathrm{T}_{m} M$. Let $E_{m}$ be a $K_{m}$-equivariant supplement to $\mathfrak{k} / \mathfrak{k}_{m}$ in $\mathrm{T}_{m} M$. Using that $\operatorname{Tr}_{\beta}\left|\mathrm{T}_{m} M\right|=$ $\operatorname{Tr}_{\beta}\left|\mathfrak{k} / \mathfrak{k}_{m}\right|+\operatorname{Tr}_{\beta}\left|E_{m}\right|$, we see that (II) is equivalent to

$$
\|\beta\|^{2}+\frac{1}{2} \operatorname{Tr}_{\beta}\left|E_{m}\right| \geq \frac{1}{2} \operatorname{Tr}_{\beta}\left|\mathfrak{k}_{m}\right| .
$$

\footnotetext{
${ }^{5} \bar{e}$ is the class of the neutral element in $W\left(K_{\rho_{\sigma}}\right) \backslash W$.
} 
Since the moment map $\Phi$ is equivariant, the Lie algebra stabilizer $\mathfrak{k}_{m}$ is contained in Lie algebra stabilizer $\mathfrak{k}_{\Phi(m)}$. Finally, we see that (I) $\Leftrightarrow(\mathbf{I I}) \Leftrightarrow$ (III) are induced by the following inequality

$$
\|\beta\|^{2} \geq \frac{1}{2} \operatorname{Tr}_{\beta}\left|\mathfrak{k}_{\Phi(m)}\right| .
$$

Lemma 4.3. - For any $(m, \xi) \in \operatorname{Cr}\left(\left\|\Phi_{N}\right\|^{2}\right)$ the vector $\beta:=\Phi(m)-\xi$ satisfies the inequality $(\boldsymbol{I V})$.

- Let $(m, \xi) \in \operatorname{Cr}\left(\left\|\Phi_{N}\right\|^{2}\right)$ such that $\beta:=\Phi(m)-\xi$ satisfies the $\|\beta\|^{2}=$ $\frac{1}{2} \boldsymbol{T r}_{\beta}\left|\mathfrak{k}_{\Phi(m)}\right|$. Then there exists a face $\tau$ of $\sigma$ such that

(1) $\mu \in \bar{\tau}$

(2) $(m, \xi)$ belongs to the $K$-orbit of $\Phi^{-1}\left(\mu+\rho-\rho_{\tau}\right) \times\{\mu+\rho\} \subset N$.

(3) $\beta$ belongs to the coadjoint orbit $K \cdot\left(-\rho_{\tau}\right)$.

Proof. Up to the multiplication of $(m, \xi)$ by an element of $K$, we can assume that $\beta \in \mathfrak{t}^{*}$. Up to the multiplication of $n=(m, \xi)$ by an element of the stabilizer subgroup $K_{\beta}:=\{k \in K \mid \operatorname{Ad}(k) \beta=\beta\}$ we can assume that $n=$ $(m, w(\mu+\rho))$ with $m \in M^{\beta}$ and $\Phi(m)=\beta+w(\mu+\rho) \in \mathfrak{t}^{*}$.

Up to the multiplication of $n=(m, w(\mu+\rho))$ by an element of the Weyl group, we can assume that $\Phi(m)$ belongs to the Weyl chamber: let $\tau$ be the face of $\sigma$ containing $\Phi(m)$ so that $K_{\Phi(m)}=K_{\tau}$.

So we have to prove that for $\Phi(m)=a \in \tau$ and $w \in W$ the vector $\beta=a-w(\mu+\rho)$ satisfies the relation

$$
\|\beta\|^{2} \geq \frac{1}{2} \operatorname{Tr}_{\beta}\left|\mathfrak{k}_{\tau}\right|
$$

The inequality (4.3) is the consequence of three basic inequalities. The first one is given by the following:

Lemma 4.4. Let $a \in \mathfrak{t}_{+}^{*}$ and $b$ in the interior of $\mathfrak{t}_{+}^{*}$. We have

$$
\|a-w b\| \geq\|a-b\|
$$

for any $w \in W$, and (4.4) is strict unless $w \in W\left(K_{a}\right)$.

Proof. In order to prove (4.4), we consider the function $\xi \in K \cdot b \longmapsto \| \xi-$ $a\left\|^{2}=\right\| a\left\|^{2}+\right\| b \|^{2}-2(\xi, a)$. The inclusion $K \cdot b \hookrightarrow \mathfrak{k}^{*}$ is the moment map relatively to the $K$-action. The function $\xi \in K \cdot b \mapsto(\xi, a)$, which is the $a$-th component of the moment map, has a unique local maximum on the coadjoint orbit $K \cdot b$ which is reached on an orbit of the stabilizer subgroup $K_{a}$ (see $\left[\mathbf{1 1}\right.$, Theorem 5]). Let $\mathfrak{r}$ be a subspace such that $\mathfrak{k}=\mathfrak{k}_{a} \oplus \mathfrak{r}$. For $X \in \mathfrak{r}$, we compute $\left(e^{X} \cdot b, a\right)=(b, a)+([X, b], a)+\frac{1}{2}([X,[X, b]], a)+o\left(\|X\|^{2}\right)$. The term $([X, b], a)$ vanishes and $([X,[X, b]], a)=-([X, b],[X, a]) \geq-$ Cst $\|X\|^{2}$ since $a, b$ belongs to the Weyl chamber and $\mathfrak{k}_{a} \cap \mathfrak{r}=\mathfrak{k}_{b} \cap \mathfrak{r}=\{0\}$. Then we have proved that the local (hence global) minimum of $k \cdot b \mapsto\|a-k \cdot b\|$ is reached on $K_{a} \cdot b$. 
Finally, we have proved that $\|a-w b\| \geq\|a-b\|$ for any $w \in W$, and that the equality $\|a-w b\|=\|a-b\|$ implies that $w b \in K_{a} \cdot b$, i.e., $w \in W\left(K_{a}\right)$.

The second inequality is

$$
\begin{aligned}
\|\mu+\rho-a\| & \geq \frac{\left(\mu+\rho-a, \rho_{\tau}\right)}{\left\|\rho_{\tau}\right\|} \\
& =\frac{1}{\left\|\rho_{\tau}\right\|} \underbrace{\left(\mu, \rho_{\tau}\right)}_{\geq 0}+\frac{1}{\left\|\rho_{\tau}\right\|} \underbrace{\left(\rho-\rho_{\tau}-a, \rho_{\tau}\right)}_{=0}+\frac{1}{\left\|\rho_{\tau}\right\|}\left(\rho_{\tau}, \rho_{\tau}\right) \\
& \geq\left\|\rho_{\tau}\right\| .
\end{aligned}
$$

Note that (4.5) is strict unless $\mu \in \bar{\tau}$ and $\mu+\rho-a=\rho_{\tau}$. The third inequality is

$$
\frac{1}{2} \operatorname{Tr}_{\beta}\left|\mathfrak{k}_{\tau}\right| \leq\left\|\rho_{\tau}\right\|\|\beta\| .
$$

See Example 3.7. If we put (4.4), (4.5) and (4.6) together we have for $\beta=$ $a-w(\mu+\rho)$ the inequalities

$$
\|\beta\|^{2} \geq\|\beta\|\|a-(\mu+\rho)\| \geq\|\beta\|\left\|\rho_{\tau}\right\| \geq \frac{1}{2} \operatorname{Tr}_{\beta}\left|\mathfrak{k}_{\tau}\right|,
$$

and the equality $\|\beta\|^{2}=\frac{1}{2} \operatorname{Tr}_{\beta}\left|\mathfrak{k}_{\tau}\right|$ holds if and only if we have the equality in (4.4), (4.5) and (4.6).

However, equalities in (4.4) and (4.5) gives that $w \in W\left(K_{\tau}\right), \mu \in \bar{\tau}$ and $a=\mu+\rho-\rho_{\tau} \in \tau$. Then $(m, w(\mu+\rho))=w\left(m^{\prime}, \mu+\rho\right)$ with $\Phi\left(m^{\prime}\right)=$ $w^{-1}\left(\mu+\rho-\rho_{\tau}\right)=\mu+\rho-\rho_{\tau}$ and $\beta=\mu+\rho-\rho_{\tau}-w(\mu+\rho)=-w \rho_{\tau}$. We have then

$$
\frac{1}{2} \operatorname{Tr}_{\beta}\left|\mathfrak{k}_{\tau}\right|=\frac{1}{2} \operatorname{Tr}_{\rho_{\tau}}\left|\mathfrak{k}_{\tau}\right|=\left\|\rho_{\tau}\right\|^{2},
$$

which is the equality in (4.6).

Since the strict inequality in (IV) implies the strict inequality in (I), Lemma 4.3 tells us that the identity (3.10) holds on $C_{\beta}$ for all $\beta \in \mathcal{B}^{\mu}$ when $\mu \notin \bar{\sigma}$. When $\mu \in \bar{\sigma}$ the identity (3.10) holds

(1) on $C_{\beta}$ for the $\beta$ which are not in $K \cdot\left(-\rho_{\tau}\right)$, where $\tau$ is a face of $\sigma$ such that $\mu \in \bar{\tau}$,

(2) on $C_{-\rho_{\sigma}, \bar{w}}$ for all the $\bar{w} \neq \bar{e}$.

The proof of Propositions 4.1 and 4.2 is completed by

Lemma 4.5. Let $\tau$ be a face of $\sigma$, distinct from $\sigma$, such that $\mu \in \bar{\tau}$. Then the identity (3.10) holds for $C_{\beta}$ for $\beta=-\rho_{\tau}$.

Proof. Let $\beta=-\rho_{\tau}$. The critical set $C_{-\rho_{\tau}}:=K\left(N^{\rho_{\tau}} \cap \Phi_{N}^{-1}\left(-\rho_{\tau}\right)\right)$ admits the decomposition $C_{-\rho_{\tau}}=\cup_{w \in W\left(K_{\tau}\right) \backslash W} C_{-\rho_{\tau}, \bar{w}}$ where

$$
C_{-\rho_{\tau}, \bar{w}}=K\left(M^{\rho_{\tau}} \cap \Phi^{-1}\left(w(\mu+\rho)-\rho_{\tau}\right) \times\{w(\mu+\tau)\}\right) .
$$

We know then from Lemma 4.3 that the strict inequality in (IV) holds on $C_{-\rho_{\tau}, \bar{w}}$ for $\bar{w} \neq \bar{e}$. 
Let us consider now the case where $m \in M^{\rho_{\tau}} \cap \Phi^{-1}\left(\mu+\rho-\rho_{\tau}\right)$. We know that the equality holds in (IV) for $(m, \mu+\rho)$. The equality in (I) for $(m, \mu+\rho)$ is then equivalently to

$$
\operatorname{Tr}_{\beta}\left|E_{m}\right|+\operatorname{Tr}_{\beta}\left|\mathfrak{k}_{\tau} / \mathfrak{k}_{m}\right|=0 .
$$

Let us prove that (4.7) can never happen. The image of $m$ by the moment map belongs to $\tau$. Then $m$ belongs to the symplectic slice $Y_{\tau} \subset M$. A neighborhood $m$ is then $K \times_{K_{\tau}} Y_{\tau}$. So the tangent space at $m$ decomposes in two manners

$$
\begin{aligned}
\mathrm{T}_{m} M & =\mathfrak{k} / \mathfrak{k}_{\tau} \oplus \mathrm{T}_{m} Y_{\tau} \\
& =\mathfrak{k} / \mathfrak{k}_{m} \oplus E_{m} .
\end{aligned}
$$

If (4.7) holds we see that $\operatorname{Tr}_{\beta}\left|\mathrm{T}_{m} Y_{\tau}\right|=\operatorname{Tr}_{\beta}\left|E_{m}\right|+\operatorname{Tr}_{\beta}\left|\mathfrak{k}_{\tau} / \mathfrak{k}_{m}\right|=0$, which means that $\beta=-\rho_{\tau}$ acts trivially on the tangent space $\mathrm{T}_{m} Y_{\tau}$. Hence, it would implies that $\rho_{\tau}$ acts trivially on the manifold $Y_{\tau}$. Since $Y_{\sigma} \subset Y_{\tau}$, the action of $\rho_{\tau}$ on the principal slice $Y_{\sigma}$ is also trivial.

We know that $\left[\mathfrak{k}_{\sigma}, \mathfrak{k}_{\sigma}\right]$ acts trivially on $Y_{\sigma}$ : since $\rho_{\sigma} \in\left[\mathfrak{k}_{\sigma}, \mathfrak{k}_{\sigma}\right]$, the infinitesimal action of $\rho_{\sigma}$ is trivial on $Y_{\sigma}$. Finally if (4.7) holds, we have that

$$
\rho_{\tau / \sigma}:=\rho_{\tau}-\rho_{\sigma} \in \mathbb{R} \sigma
$$

acts trivially on $Y_{\sigma}$. Note that $\rho_{\tau / \sigma}$ is a sum of weights, which are orthogonal to $\tau$.

The moment polytope of $M, \Delta(M)$, which is equal to the closure of $\Phi\left(Y_{\sigma}\right) \subset \sigma$ is a convex polytope. Since the action of $\rho_{\tau / \sigma}$ is trivial on $Y_{\sigma}$ we knows that the map $\xi \in \Delta(M) \mapsto\left(\xi, \rho_{\tau / \sigma}\right)$ is constant.

Finally, we can use the last information in our hands: $\mu+\rho-\rho_{\tau}=\Phi(m)$ belongs to $\Delta(M)$. Then for $\xi \in \Delta(M)$ we have

$$
\left(\xi, \rho_{\tau / \sigma}\right)=\left(\mu+\rho-\rho_{\tau}, \rho_{\tau / \sigma}\right)=0,
$$

since $\mu+\rho-\rho_{\tau} \in \tau$ and $\rho_{\tau / \sigma} \in \tau^{\perp}$. It is contradictory with the fact that $\left(\xi, \rho_{\tau / \sigma}\right)=\left(\xi, \rho_{\tau}\right)>0$ for any $\xi \in \sigma$.

We have finally proved that when $(m, \xi) \in N^{\rho_{\tau}} \cap \Phi_{N}^{-1}\left(-\rho_{\tau}\right)$ the vector $\beta=\Phi(m)-\xi$ satisfies $\|\beta\|^{2}+\frac{1}{2} \operatorname{Tr}_{\beta}\left|\mathrm{T}_{m} M\right|>\operatorname{Tr}_{\beta}|\mathfrak{k}|$.

4.2. Computation of the multiplicities when $\boldsymbol{\sigma}=\mathfrak{t}_{+}^{*}$. In this section, we suppose that the moment polytope $\Delta(M)=\Phi(M) \cap \mathfrak{t}_{+}^{*}$ intersects the interior of the Weyl chamber. Let $\Delta(M)^{o} \subset\left(\mathfrak{t}_{+}^{*}\right)^{o}$ be the relative interior of the moment polytope. We know that $\mathrm{m}_{\mu}=\left[\mathcal{Q}_{0}^{K}\left(M \times \overline{\mathcal{O}_{\mu}}\right)\right]^{K}$ for any $\mu \in \widehat{K}$. In Definition 2.11, we have defined the number $\mathcal{Q}\left(M_{\mu}^{\mathrm{t}_{+}^{*}}\right)$ has follows. If $\mu+\rho \notin$ $\Delta(M)^{\mathrm{o}}$, we set $\mathcal{Q}\left(M_{\mu}^{\mathrm{t}_{+}^{*}}\right)=0$. If $\mu+\rho \in \Delta(M)^{\mathrm{o}}$, we consider, for $\varepsilon$ generic and small enough, the orbifold reduced space $M_{\mu+\varepsilon}^{\mathfrak{t}_{+}^{*}}:=\Phi^{-1}(\mu+\varepsilon+\rho) / T$ 
and the orbifold line bundle

$$
\tilde{\mathcal{L}}_{\mu+\varepsilon}=\left(\left.\tilde{L}\right|_{\Phi^{-1}(\mu+\varepsilon+\rho)} \otimes \mathbb{C}_{-\mu}\right) / T .
$$

The Spin-quantization $\mathcal{Q}_{\text {spin }}\left(M_{\mu}^{\mathfrak{t}_{+}^{*}}\right) \in \mathbb{Z}$ is defined as the Riemann-Roch number

$$
R R\left(M_{\mu+\varepsilon}^{\mathfrak{t}_{+}^{*}}, \tilde{\mathcal{L}}_{\mu+\varepsilon}\right) .
$$

The main result of this section is the following

Theorem 4.6. The number $\left[\mathcal{Q}_{0}^{K}\left(M \times \overline{\mathcal{O}_{\mu}}\right)\right]^{K}$ is equal to $\mathcal{Q}_{\text {spin }}\left(M_{\mu}^{\mathfrak{t}_{+}^{*}}\right)$.

Proof. When $\mu+\rho \notin \Delta(M)$, we see that $\mathcal{Q}_{0}^{K}\left(M \times \overline{\mathcal{O}_{\mu}}\right)=0$ since the moment map on $M \times \overline{\mathcal{O}_{\mu}}$ does not goes through $0 \in \mathfrak{k}^{*}$. We have then $\left[\mathcal{Q}_{0}^{K}(M \times\right.$ $\left.\left.\overline{\mathcal{O}_{\mu}}\right)\right]^{K}=\mathcal{Q}_{\text {spin }}\left(M_{\mu}^{\mathfrak{t}_{+}^{*}}\right)=0$.

We consider now a dominant weight $\mu$ such that $\mu+\rho \in \Delta(M)$. Let $Y=$ $\Phi^{-1}\left(\left(\mathfrak{t}_{+}^{*}\right)^{\circ}\right)$ be the symplectic slice with its canonical symplectic form $\omega_{Y}$. The action of $T$ on $\left(Y, \omega_{Y}\right)$ is Hamiltonian with moment map $\Phi_{Y}:=\left.\Phi\right|_{Y}-\rho$. We know that $\left.\tilde{L}\right|_{Y}$ Spin-prequantizes $\left(Y, \omega_{Y}, \Phi_{Y}\right)$ (see Lemma 2.8).

We consider the Riemann-Roch character $R_{0}^{T}\left(Y,\left.\tilde{L}\right|_{Y} \otimes \mathbb{C}_{-\mu}\right)$ which is localized near $\left(\Phi_{Y}-\mu\right)^{-1}(0) \subset Y$. Thanks to the induction formula (3.11), we know that

$$
\begin{aligned}
\mathrm{m}_{\mu}=\left[\mathcal{Q}_{0}^{K}\left(M \times \overline{\mathcal{O}_{\mu}}\right)\right]^{K} & =\left[R R_{0}^{K}\left(M \times \overline{\mathcal{O}_{\mu}}, \tilde{L} \otimes \mathbb{C}_{[-\mu]}\right)\right]^{K} \\
& =\left[R R_{0}^{T}\left(Y,\left.\tilde{L}\right|_{Y} \otimes \mathbb{C}_{-\mu}\right)\right]^{\mathrm{T}} \\
& =\left[R R_{\Phi_{Y}^{T}-\mu}\left(U,\left.\tilde{L}\right|_{U} \otimes \mathbb{C}_{-\mu}\right)\right]^{\mathrm{T}}
\end{aligned}
$$

where $U$ is a small neighborhood of $\Phi_{Y}^{-1}(\mu)$ in $Y$.

The computation of the expression $\left[R R_{\Phi_{Y}-\mu}^{T}\left(U,\left.\tilde{L}\right|_{U} \otimes \mathbb{C}_{-\mu}\right)\right]^{\mathrm{T}}$ is identical to what we have done in Section 3.4. For $\varepsilon$ small enough and generic, we get

$$
\begin{aligned}
{\left[R R_{\Phi_{Y}-\mu}^{T}\left(U,\left.\tilde{L}\right|_{U} \otimes \mathbb{C}_{-\mu}\right)\right]^{T} } & =\left[R R_{\Phi_{Y}-\mu-\varepsilon}^{T}\left(U,\left.\tilde{L}\right|_{U} \otimes \mathbb{C}_{-\mu}\right)\right]^{T} \\
& =R R\left(M_{\mu+\varepsilon}^{\mathfrak{t}_{+}^{*}}, \tilde{\mathcal{L}}_{\mu+\varepsilon}\right) \\
& =\mathcal{Q}_{\operatorname{spin}}\left(M_{\mu}^{\mathfrak{t}_{+}^{*}}\right) .
\end{aligned}
$$

When $\mu+\rho$ does not belong to the relative interior of $\Delta(M)$, we can choose $\varepsilon$ so that $\mu+\rho+\varepsilon \notin \Delta(M)$, and then $R R\left(M_{\mu+\varepsilon}^{\mathfrak{t}_{+}^{*}}, \tilde{\mathcal{L}}_{\mu+\varepsilon}\right)=$ $\mathcal{Q}_{\text {spin }}\left(M_{\mu}^{\mathfrak{t}_{+}^{*}}\right)=0$. 
4.3. Computation of the multiplicities when $\boldsymbol{\sigma} \neq \mathfrak{t}_{+}^{*}$. Let $\mu \in \bar{\sigma}$ so that $\mu+\rho-\rho_{\sigma} \in \sigma$. In the rest of this section the term $\beta$ is $-\rho_{\sigma}$.

We have proved in the previous section that $\mathrm{m}_{\mu}=\left[\mathcal{Q}_{\beta, \bar{e}}^{K}\left(M \times \overline{\mathcal{O}_{\mu}}\right)\right]^{K}$ where the character $\mathcal{Q}_{\beta, \bar{e}}^{K}\left(M \times \overline{\mathcal{O}_{\mu}}\right)$ corresponds to the Riemann-Roch character $R R_{\beta, \bar{e}}^{K}\left(N, \tilde{L}_{N}\right)$ localized with the Kirwan vector field near

$$
C_{\beta, \bar{e}}=K\left(M^{\beta} \cap \Phi^{-1}\left(\mu+\rho-\rho_{\sigma}\right) \times\{\mu+\rho\}\right) \subset \operatorname{Cr}\left(\left\|\Phi_{N}\right\|^{2}\right) .
$$

Look now at $N$ as a $K_{\beta}$-Hamiltonian manifold. Let $\Phi_{N}^{\prime}: N \rightarrow \mathfrak{k}_{\beta}^{*}$ be the corresponding moment map. We are interested in the component ${ }^{6}$

$$
\begin{aligned}
C_{\beta}^{\prime} & :=K_{\beta}\left(N^{\beta} \cap\left(\Phi_{N}^{\prime}\right)^{-1}(\beta)\right) \\
& =N^{\beta} \cap \Phi_{N}^{-1}(\beta) \\
& =\bigcup_{\bar{w} \in W\left(K_{\beta}\right) \backslash W} K_{\beta}\left(M^{\beta} \cap \Phi^{-1}\left(w(\mu+\rho)-\rho_{\sigma}\right) \times\{w(\mu+\rho)\}\right)
\end{aligned}
$$

of the critical set $\operatorname{Cr}\left(\left\|\Phi_{N}^{\prime}\right\|^{2}\right)$. Let us consider the Riemann-Roch character

$$
R R_{\beta, \bar{e}}^{K_{\beta}}(N,-)
$$

localized with the Kirwan vector field near

$$
C_{\beta, \bar{e}}^{\prime}:=K_{\beta}\left(M^{\beta} \cap \Phi^{-1}\left(\mu+\rho-\rho_{\sigma}\right) \times\{\mu+\rho\}\right) \subset C_{\beta}^{\prime} .
$$

We have proved in $[\mathbf{2 1}$, Th. 6.16, Cor. 6.17] that

$$
R R_{\beta, \bar{e}}^{K}\left(N, \tilde{L}_{N}\right)=\operatorname{Ind}_{K_{\beta}}^{K}\left(R R_{\beta, \bar{e}}^{K_{\beta}}\left(N, \tilde{L}_{N}\right) \wedge_{\mathbb{C}}^{\bullet}\left(\mathfrak{k} / \mathfrak{k}_{\beta}\right)_{\mathbb{C}}\right),
$$

where $\operatorname{Ind}_{K_{\beta}}^{K}: R^{-\infty}\left(K_{\beta}\right) \rightarrow R^{-\infty}(K)$ is the induction map, and $\left(\mathfrak{k} / \mathfrak{k}_{\beta}\right)_{\mathbb{C}}$ is the complexification of the real $K_{\beta}$-module $\mathfrak{k} / \mathfrak{k}_{\beta}$. It gives that

$$
\mathrm{m}_{\mu}=\left[R R_{\beta, \bar{e}}^{K}\left(N, \tilde{L}_{N}\right)\right]^{K}=\left[R R_{\beta, \bar{e}}^{K_{\beta}}\left(N, \tilde{L}_{N}\right) \wedge_{\mathbb{C}}\left(\mathfrak{k} / \mathfrak{k}_{\beta}\right) \mathbb{C}\right]^{K_{\beta}}
$$

Let $Y_{\sigma}$ be the principal symplectic slice of $M$. Recall that the subgroup $\left[K_{\sigma}, K_{\sigma}\right]$ acts trivially on $Y_{\sigma}$ and that $\beta=-\rho_{\sigma}$ belongs to $\left[\mathfrak{k}_{\sigma}, \mathfrak{k}_{\sigma}\right]$ : hence $\Phi^{-1}\left(\mu+\rho-\rho_{\sigma}\right) \subset Y_{\sigma} \subset M^{\beta}$ and then

$$
C_{\beta, \bar{e}}^{\prime}=K_{\beta}\left(\Phi^{-1}\left(\mu+\rho-\rho_{\sigma}\right) \times\{\mu+\rho\}\right) .
$$

We are looking at a $K_{\beta}$-invariant neighborhood $\mathcal{U}$ of $C_{\beta, \bar{e}}^{\prime}$ in $N^{\beta}$. We consider the open neighborhood $K \times_{K_{\sigma}} Y_{\sigma}$ of $\Phi^{-1}\left(\mu+\rho-\rho_{\sigma}\right)$ in $M$. Since $K_{\beta} \cap K_{\sigma}=T$, one sees that

$$
K_{\beta} \times_{T} Y_{\sigma} \subset\left(K \times_{K_{\sigma}} Y_{\sigma}\right)^{\beta}
$$

\footnotetext{
${ }^{6}$ In the second equality, we use that $\beta$ is central in $\mathfrak{k}_{\beta}$, and that $\Phi_{N}^{\prime}=\Phi_{N}$ on $N^{\beta}$.
} 
is a $K_{\beta}$-invariant neighborhood of $Y_{\sigma}$ in $M^{\beta}$. Then we can take

$$
\mathcal{U}:=\left(K_{\beta} \times_{T} Y_{\sigma}\right) \times K_{\beta}(\mu+\rho) \subset N^{\beta} .
$$

We look at $\mathcal{U}$ as a Hamiltonian $K_{\beta}$-manifold with moment map $\Phi_{\mathcal{U}}([k, y]$, $\xi)=k \Phi(y)-\xi \in \mathfrak{k}_{\beta}^{*}$. The set $C_{\beta, \bar{e}}^{\prime}$ is a connected component of critical points of $\operatorname{Cr}\left(\left\|\Phi_{\mathcal{U}}\right\|^{2}\right)$, and we consider the Riemann-Roch character

$$
R R_{\beta}^{K_{\beta}}(\mathcal{U},-)
$$

localized with the Kirwan vector field near $C_{\beta, \bar{e}}^{\prime} \subset \mathcal{U}$.

Let $\mathcal{N}$ be the normal bundle of $\mathcal{U}$ in $N$. We have $\mathcal{N}=\mathcal{N}_{1} \otimes \mathcal{N}_{2}$ where $\mathcal{N}_{1}$ is the normal bundle of $K_{\beta} \times_{T} Y_{\sigma}$ in $K \times_{K_{\sigma}} Y_{\sigma}$ and $\mathcal{N}_{2}$ is the normal bundle of $K_{\beta}(\mu+\rho)$ in $K(\mu+\rho)$. One computes that $\mathcal{N}_{1}=K_{\beta} \times_{T} N_{1}$ where

$$
N_{1}=\sum_{\substack{\alpha>\left.0 \\ \alpha\right|_{\sigma} \neq 0,(\alpha, \beta) \neq 0}} \mathfrak{k}(\alpha),
$$

and that $\mathcal{N}_{2}=K_{\beta} \times_{T} N_{2}$ where

$$
N_{2}=\sum_{\substack{\alpha<0 \\(\alpha, \beta) \neq 0}} \mathfrak{k}(\alpha)
$$

We decompose $\mathcal{N}$ in the sum of the polarized bundle $\mathcal{N}^{+, \beta}$ and $\mathcal{N}^{-, \beta}$. Similarly let $\mathcal{N}_{\mathbb{C}}$ the complexified bundle, and its polarized $\beta$-positive part $\mathcal{N}_{\mathbb{C}}^{+, \beta}$.

Let $S\left(\mathcal{N}_{\mathbb{C}}^{+, \beta}\right)=\sum_{k \geq 0} S^{k}\left(\mathcal{N}_{\mathbb{C}}^{+, \beta}\right)$ be the symmetric algebra vector bundle associated to $\mathcal{N}_{\mathbb{C}}^{+, \beta}$. Let us compute the rank $n_{+, \beta}$ of the polarized vector bundle vector $\mathcal{N}^{+, \beta}$. We have

$$
\begin{aligned}
n_{+, \beta} & =\sharp\left\{\alpha>0 \mid(\alpha, \beta)>0 \text { and }\left.\alpha\right|_{\sigma} \neq 0\right\}+\sharp\{\alpha<0 \mid(\alpha, \beta)>0\} \\
& =\sharp\{\alpha>0 \mid(\alpha, \beta)>0\}+\sharp\{\alpha<0 \mid(\alpha, \beta)>0\} \\
& =\frac{1}{2} \operatorname{dim}\left(K / K_{\beta}\right) .
\end{aligned}
$$

In (1) we use that $\left.\alpha\right|_{\sigma}=0$ imposes $\left(\alpha, \rho-\rho_{\sigma}\right)=0$. Then $(\alpha, \beta)=$ $-(\alpha, \rho)<0$ for $\alpha>0$. Let $\operatorname{det} \mathcal{N}^{+, \beta}$ be the determinant line bundle associated to $\mathcal{N}^{+, \beta}$.

Thanks to the results in $[\mathbf{2 1}$, Section 6.3], we know that

$$
R R_{\beta, e}^{K_{\beta}}\left(N, \tilde{L}_{N}\right)=(-1)^{n_{+, \beta}} R R_{\beta}^{K_{\beta}}\left(\mathcal{U},\left.\tilde{L}_{N}\right|_{\mathcal{U}} \otimes \operatorname{det} \mathcal{N}^{+, \beta} \otimes S\left(\mathcal{N}_{\mathbb{C}}^{+, \beta}\right)\right)
$$


Hence we know that $\mathrm{m}_{\mu}=\left[R R_{\beta, \bar{e}}^{K}\left(N, \tilde{L}_{N}\right)\right]^{K}$ is equal to $(-1)^{n_{+, \beta}}$ times

$$
\left[R R_{\beta}^{K_{\beta}}\left(\mathcal{U}, \tilde{L}_{N} \mid \mathcal{U} \otimes \operatorname{det} \mathcal{N}^{+, \beta} \otimes S\left(\mathcal{N}_{\mathbb{C}}^{+, \beta}\right)\right) \wedge_{\mathbb{C}}^{\bullet}\left(\mathfrak{k} / \mathfrak{k}_{\beta}\right)_{\mathbb{C}}\right]^{K_{\beta}}
$$

$$
=\sum_{k \geq 0}\left[R R_{\beta}^{K_{\beta}}\left(\mathcal{U},\left.\tilde{L}_{N}\right|_{\mathcal{U}} \otimes \operatorname{det} \mathcal{N}^{+, \beta} \otimes S^{k}\left(\mathcal{N}_{\mathbb{C}}^{+, \beta}\right)\right) \wedge_{\mathbb{C}}\left(\mathfrak{k} / \mathfrak{k}_{\beta}\right)_{\mathbb{C}}\right]^{K_{\beta}} .
$$

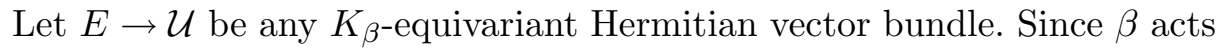
trivially on $\mathcal{U}$ we can look at the Lie derivative $\mathcal{L}^{E}(\beta)$ on $E$. Then $\frac{1}{i} \mathcal{L}^{E}(\beta)$ defines for each $x \in \mathcal{U}$ a Hermitian endomorphism of $E_{x}$. Let us denote introduce Tian-Zhang's positivity condition (see (4.2) in $[\mathbf{2 7}]$ ): we write

$$
\frac{1}{i} \mathcal{L}^{E}(\beta)>0
$$

when all its eigenvalue on the fibers of $E$ are strictly positive.

We made in [21, Lemma 9.4] the crucial observation

Lemma 4.7. If $\frac{1}{i} \mathcal{L}^{E}(\beta)>0$, then $\left[R R_{\beta}^{K_{\beta}}(\mathcal{U}, E)\right]^{K_{\beta}}=0$.

Let us compute the Lie action $\mathcal{L}(\beta)$ on the fibers of the bundle $\left.\tilde{L}_{N}\right|_{\mathcal{U}} \otimes$ $\operatorname{det} \mathcal{N}^{+, \beta} \otimes S^{k}\left(\mathcal{N}_{\mathbb{C}}^{+, \beta}\right)$. It is easy to check (see $\left.[\mathbf{2 2}]\right)$ that on $\left.\tilde{L}_{N}\right|_{\mathcal{U}} \otimes \operatorname{det} \mathcal{N}^{+, \beta}$ the Lie action $\frac{1}{i} \mathcal{L}(\beta)$ is equal to

$$
\|\beta\|^{2}+\frac{1}{2} \operatorname{Tr}_{\beta}|\mathcal{N}|
$$

Look now at the Lie derivative $\mathcal{L}(\beta)$ on $\wedge_{\mathbb{C}}^{\bullet}\left(\mathfrak{k} / \mathfrak{k}_{\beta}\right)_{\mathbb{C}}$. As a $T$-module $\wedge_{\mathbb{C}}^{\bullet}\left(\mathfrak{k} / \mathfrak{k}_{\beta}\right)_{\mathbb{C}}$ is equal to

$$
\begin{aligned}
\prod_{(\alpha, \beta) \neq 0}\left(1-\mathrm{e}^{\mathrm{i} \alpha}\right) & =\prod_{(\alpha, \beta)<0}\left(1-\mathrm{e}^{\mathrm{i} \alpha}\right) \prod_{(\alpha, \beta)>0}\left(1-\mathrm{e}^{\mathrm{i} \alpha}\right) \\
& =(-1)^{1 / 2 \operatorname{dim}\left(K / K_{\beta}\right)} \mathrm{e}^{-\mathrm{i} \delta_{\beta}}\left(\prod_{(\alpha, \beta)>0}\left(1-\mathrm{e}^{\mathrm{i} \alpha}\right)\right)^{2}
\end{aligned}
$$

with $\delta_{\beta}=\sum_{(\alpha, \beta)>0} \alpha$. Note that $\mathrm{e}^{-\mathrm{i} \delta_{\beta}}$ defines a character of the group $K_{\beta}$ that we denote $\mathbb{C}_{-\delta_{\beta}}$. We have proved then that

$$
\wedge_{\mathbb{C}}^{\bullet}\left(\mathfrak{k} / \mathfrak{k}_{\beta}\right)_{\mathbb{C}}=(-1)^{n_{+, \beta}} \mathbb{C}_{-\delta_{\beta}} \oplus R
$$

where the Lie derivative $\frac{1}{i} \mathcal{L}(\beta)$ on $\mathbb{C}_{-\delta_{\beta}}$ is equal to $-\left(\delta_{\beta}, \beta\right)=-\operatorname{Tr}_{\beta}|\mathfrak{k}|$ and the Lie derivative $\frac{1}{i} \mathcal{L}(\beta)$ on the $\mathfrak{k}_{\beta}$-module $R$ is $>-\operatorname{Tr}_{\beta}|\mathfrak{k}|$.

Since $\|\beta\|^{2}+\frac{1}{2} \operatorname{Tr}_{\beta}|\mathcal{N}|=\operatorname{Tr}_{\beta}|\mathfrak{k}|$, we can conclude that the Lie derivative $\frac{1}{i} \mathcal{L}(\beta)$

(1) is equal to zero on $\left.\tilde{L}_{N}\right|_{\mathcal{U}} \otimes \operatorname{det} \mathcal{N}^{+, \beta} \otimes \mathbb{C}_{-\delta_{\beta}}$,

(2) is $>0$ on $\tilde{L}_{N} \mid \mathcal{U} \otimes \operatorname{det} \mathcal{N}^{+, \beta} \otimes R$, 
(3) is $>0$ on $\tilde{L}_{N} \mid \mathcal{U} \otimes \operatorname{det} \mathcal{N}^{+, \beta} \otimes S^{k}\left(\mathcal{N}_{\mathbb{C}}^{+, \beta}\right) \otimes \wedge_{\mathbb{C}}\left(\mathfrak{k} / \mathfrak{k}_{\beta}\right)_{\mathbb{C}}$ for any $k \geq 1$.

With Lemma 4.7, we see that the sum (4.10) restricts to

$$
(-1)^{n_{+, \beta}}\left[R R_{\beta}^{K_{\beta}}\left(\mathcal{U},\left.\tilde{L}_{N}\right|_{\mathcal{U}} \otimes \operatorname{det} \mathcal{N}^{+, \beta}\right) \otimes \mathbb{C}_{-\delta_{\beta}}\right]^{K_{\beta}} .
$$

At this stage we have proved that the multiplicity $\mathrm{m}_{\mu}$ is equal to

$$
\left[R R_{\beta}^{K_{\beta}}\left(\mathcal{U},\left.\tilde{L}_{N}\right|_{\mathcal{U}} \otimes \operatorname{det} \mathcal{N}^{+, \beta}\right) \otimes \mathbb{C}_{-\delta_{\beta}}\right]^{K_{\beta}} .
$$

On the symplectic slice $\left(Y_{\sigma}, \omega_{\sigma}\right)$, we have the moment map $\Phi_{\sigma}-\mu$ relative to the action of $Z_{\sigma}$. The data $\left(Y_{\sigma}, \omega_{\sigma}, \Phi_{\sigma}-\mu\right)$ is Spin-prequantized by the line bundle $\left.\tilde{L}\right|_{Y_{\sigma}} \otimes \mathbb{C}_{-\mu}$. Let

$$
R R_{0}^{Z_{\sigma}}\left(Y_{\sigma},\left.\tilde{L}\right|_{Y_{\sigma}} \otimes \mathbb{C}_{-\mu}\right) \in R^{-\infty}\left(Z_{\sigma}\right)
$$

be the Riemann-Roch character localized near $\left(\Phi_{\sigma}-\mu\right)^{-1}(0)=\Phi^{-1}(\mu+\rho-$ $\left.\rho_{\sigma}\right) \subset Y_{\sigma}$.

We conclude the computation of the multiplicity $\mathrm{m}_{\mu}$ with the

Lemma 4.8. We have

$$
\begin{aligned}
\mathrm{m}_{\mu} & =\left[R R_{\beta}^{K_{\beta}}\left(\mathcal{U},\left.\tilde{L}_{N}\right|_{\mathcal{U}} \otimes \operatorname{det} \mathcal{N}^{+, \beta}\right) \otimes \mathbb{C}_{-\delta_{\beta}}\right]^{K_{\beta}} \\
& =\left[R R_{0}^{Z_{\sigma}}\left(Y_{\sigma},\left.\tilde{L}\right|_{Y_{\sigma}} \otimes \mathbb{C}_{-\mu}\right)\right]^{Z_{\sigma}} \\
& =\mathcal{Q}_{\text {spin }}\left(M_{\mu}^{\sigma}\right) .
\end{aligned}
$$

Proof. Let us prove that (1) is a consequence of the induction formula of Proposition 3.10. First, we notice that the data $\left(Y_{\sigma}, \omega_{\sigma}, \Phi_{\sigma}-\mu,\left.\tilde{L}\right|_{Y_{\sigma}} \otimes \mathbb{C}_{-\mu}\right)$ is naturally equipped with an action of the maximal torus, but with a trivial action of $T / Z_{\sigma}$. So the generalized character (4.12) coincides with

$$
R R_{0}^{T}\left(Y_{\sigma},\left.\tilde{L}\right|_{Y_{\sigma}} \otimes \mathbb{C}_{-\mu}\right) \in R^{-\infty}(T) .
$$

Let us consider the Hamiltonian $K_{\beta}$-manifold $\mathcal{U}:=\left(K_{\beta} \times_{T} Y_{\sigma}\right) \times$ $\overline{K_{\beta}(\mu+\rho)}$. Since $K_{\beta}$ acts trivially on $\rho_{\sigma}$ the map $\xi \mapsto \xi-\rho_{\sigma}$ realizes a $K_{\beta}$-equivariant symplectomorphic between the coadjoint orbits $\overline{K_{\beta}(\mu+\rho)}$ and

$$
\overline{\mathcal{O}}:=\overline{K_{\beta}\left(\mu+\rho-\rho_{\sigma}\right)} \text {. }
$$

The manifold $\mathcal{U}$ is then symplectomorphic to $\left(K_{\beta} \times_{T} Y_{\sigma}\right) \times \overline{\mathcal{O}}$. Moreover, one sees that the generalized Riemann-Roch character $R R_{\beta}^{K_{\beta}}(\mathcal{U},-)$ coincides with the Riemann-Roch character

$$
R R_{0}^{K_{\beta}}\left(\left(K_{\beta} \times_{T} Y_{\sigma}\right) \times \overline{\mathcal{O}},-\right)
$$

localized on $C_{0}:=K_{\beta}\left(\Phi^{-1}\left(\mu+\rho-\rho_{\sigma}\right) \times\left\{\mu+\rho-\rho_{\sigma}\right\}\right)$.

Since $K_{\beta} \cap K_{\sigma}=T$, the Hamiltonian $T$-manifold $Y_{\sigma}$ corresponds to the symplectic slice of the Hamiltonian $K_{\beta}$-manifold $K_{\beta} \times_{T} Y_{\sigma}$. 
The bundle $\operatorname{det} \mathcal{N}^{+, \beta}$ over $\left(K_{\beta} \times_{T} Y_{\sigma}\right) \times \overline{\mathcal{O}}$ is equal to the product of $K_{\beta} \times_{T} \mathbb{C}_{\delta_{1}} \rightarrow K_{\beta} \times_{T} Y_{\sigma}$ with $K_{\beta} \times_{T} \mathbb{C}_{\delta_{2}} \rightarrow \overline{\mathcal{O}}$, where

$$
\delta_{1}=\sum_{\substack{\alpha>0 \\(\alpha, \beta)>0}} \alpha \text { and } \delta_{2}=\sum_{\substack{\alpha<0 \\(\alpha, \beta)>0}} \alpha .
$$

The line bundle $\tilde{L}_{N}$ is equal to the product of $\tilde{L}$ with $K \times_{T} \mathbb{C}_{-\mu}$. Then the restrictions of the lines bundle $\operatorname{det} \mathcal{N}^{+, \beta}$ and $\tilde{L}_{N}$ to $Y_{\sigma} \times\left\{\mu+\rho-\rho_{\sigma}\right\}$ are respectively equal to, the trivial line bundle $\mathbb{C}_{\delta_{1}+\delta_{2}}=\mathbb{C}_{\delta_{\beta}}$, and to the line bundle $\left.\tilde{L}\right|_{Y_{\sigma}} \otimes \mathbb{C}_{-\mu}$.

Finally, the induction formula of Proposition 3.10 gives that

$$
\begin{aligned}
R R_{\beta}^{K_{\beta}}\left(\mathcal{U},\left.\tilde{L}_{N}\right|_{\mathcal{U}} \otimes \operatorname{det} \mathcal{N}^{+, \beta}\right) & =R R_{0}^{K_{\beta}}\left(\left(K_{\beta} \times_{T} Y_{\sigma}\right) \times \overline{\mathcal{O}},\left.\tilde{L}_{N}\right|_{\mathcal{U}} \otimes \operatorname{det} \mathcal{N}^{+, \beta}\right) \\
& =\operatorname{Ind}_{T}^{K_{\beta}}\left(R R_{0}^{T}\left(Y_{\sigma},\left.\tilde{L}\right|_{Y_{\sigma}} \otimes \mathbb{C}_{-\mu}\right) \otimes \mathbb{C}_{\delta_{\beta}}\right) \\
& =\operatorname{Ind}_{T}^{K_{\beta}}\left(R R_{0}^{T}\left(Y_{\sigma},\left.\tilde{L}\right|_{Y_{\sigma}} \otimes \mathbb{C}_{-\mu}\right)\right) \otimes \mathbb{C}_{\delta_{\beta}}
\end{aligned}
$$

Hence

$$
\begin{aligned}
{\left[R R_{\beta}^{K_{\beta}}\left(\mathcal{U},\left.\tilde{L}_{N}\right|_{\mathcal{U}} \otimes \operatorname{det} \mathcal{N}^{+, \beta}\right) \otimes \mathbb{C}_{-\delta_{\beta}}\right]^{K_{\beta}} } & =\left[R R_{0}^{T}\left(Y_{\sigma},\left.\tilde{L}\right|_{Y_{\sigma}} \otimes \mathbb{C}_{-\mu}\right)\right]^{T} \\
& =\left[R R_{0}^{Z_{\sigma}}\left(Y_{\sigma},\left.\tilde{L}\right|_{Y_{\sigma}} \otimes \mathbb{C}_{-\mu}\right)\right]^{Z_{\sigma}} .
\end{aligned}
$$

Equality (2), i.e.,

$$
\left[R R_{0}^{Z_{\sigma}}\left(Y_{\sigma},\left.\tilde{L}\right|_{Y_{\sigma}} \otimes \mathbb{C}_{-\mu}\right)\right]^{Z_{\sigma}}=\mathcal{Q}_{\text {spin }}\left(M_{\mu}^{\sigma}\right)
$$

has been proved in Section 3.4.

\section{References}

[1] M.F. Atiyah, Elliptic operators and compact groups, Springer, 1974. Lecture notes in Mathematics, 401.

[2] M.F. Atiyah and G.B. Segal, The index of elliptic operators II, Ann. Math. 87 (1968), $531-545$.

[3] M.F. Atiyah and I.M. Singer, The index of elliptic operators I, Ann. Math. 87 (1968), $484-530$.

[4] M.F. Atiyah and I.M. Singer, The index of elliptic operators III, Ann. Math. 87 (1968), 546-604.

[5] M.F. Atiyah and I.M. Singer, The index of elliptic operators IV, Ann. Math. 93 (1971), 139-141.

[6] N. Berline, E. Getzler, and M. Vergne, Heat kernels and Dirac operators, Grundlehren, vol. 298, Springer, Berlin, 1991.

[7] N. Berline and M. Vergne, The Chern character of a transversally elliptic symbol and the equivariant index, Invent. Math. 124 (1996), 11-49. 
[8] N. Berline and M. Vergne, L'indice équivariant des opérateurs transversalement elliptiques, Invent. Math. 124 (1996), 51-101.

[9] A. Cannas da Silva, Y. Karshon and S. Tolman, Quantization of presymplectic manifolds and circle actions, Trans. Amer. Math. Soc. 352 (2000), 525-552.

[10] J. J. Duistermaat, The heat equation and the Lefschetz fixed point formula for the Spin $^{c}$-Dirac operator, Progress in Nonlinear Differential Equation and Their Applications, vol. 18, Birkhauser, Boston, 1996.

[11] V. Guillemin and S. Sternberg, Convexity properties of the moment mapping, Invent. Math. 67 (1982), 491-513.

[12] V. Guillemin and S. Sternberg, Geometric quantization and multiplicities of group representations, Invent. Math., 67 (1982), 515-538.

[13] V. Guillemin and S. Sternberg, A normal form for the moment map, in Differential Geometric Methods in Mathematical Physics (S. Sternberg, ed.), Reidel Publishing Company, Dordrecht, 1984.

[14] L. Jeffrey and F. Kirwan, Localization and quantization conjecture, Topology 36 (1997), 647-693.

[15] B. Kostant, Quantization and unitary representations, in Modern Analysis and Applications, Lecture Notes in Math., 170, Springer-Verlag, 1970, 87-207.

[16] H. Lawson and M.-L. Michelsohn, Spin geometry, Princeton Math. Series, 38. Princeton University Press, Princeton, 1989.

[17] E. Lerman, E. Meinrenken, S. Tolman and C. Woodward, Non-Abelian convexity by symplectic cuts, Topology, 37, 1998, 245-259.

[18] E. Meinrenken, Symplectic surgery and the Spin ${ }^{c}$-Dirac operator, Adv. Math. 134, (1998), 240-277.

[19] E. Meinrenken and R. Sjamaar, Singular reduction and quantization, Topology 38 (1999), 699-762.

[20] P-E. Paradan, Formules de localisation en cohomologie équivariante, Composit. Math. 117 (1999), 243-293.

[21] P-E. Paradan, Localization of the Riemann-Roch character, J. Funct. Anal. 187 (2001), 442-509.

[22] P-E. Paradan, Spin ${ }^{c}$ quantization and the $K$-multiplicities of the discrete series, Annal. Sci. N. S. 36 (2003), 805-845.

[23] P-E. Paradan and M. Vergne, Index of transversally elliptic operators, Astérique, Soc. Math. Fr. 328 (2009), 297-338.

[24] R. Sjamaar, Symplectic reduction and Riemann-Roch formulas for multiplicities, Bull. Amer. Math. Soc. 33 (1996), 327-338.

[25] J.M. Souriau, Structure des systèmes dynamiques, Maîtrise de mathématiques, Dunod, 1970.

[26] C. Teleman, The quantization conjecture revisited, Annal. Math. 152 (2000), 1-43.

[27] Y. Tian and W. Zhang, An analytic proof of the geometric quantization conjecture of Guillemin-Sternberg, Invent. Math. 132 (1998), 229-259.

[28] M. Vergne, Multiplicity formula for geometric quantization, Part I, Part II, and Part III, Duke Math. J. 82 (1996), 143-179, 181-194, 637-652.

[29] M. Vergne, Quantification géométrique et réduction symplectique, Séminaire Bourbaki 888, 2001. 
[30] N.M.J. Woodhouse, Geometric quantization, 2nd edn., Oxford Mathematical Monographs. Clarendon Press, Oxford, 1997.

Institut de Mathématiques et de Modélisation de Montpellier (I3M), UniverSITÉ MontPellier 2

E-mail address: Paul-Emile.Paradan@math.univ-montp2.fr

Received 03/04/2010, accepted 09/12/2011 